\title{
Postconditioning with Sevoflurane or Propofol Alleviate Lipopolysaccharide-Induced Neuroinflammation, but Exert Dissimilar Effects on NR2B Subunit and Cognition
}

Hongliang Liu ( $\square$ liuhl75@163.com )

Chongqing University Cancer Hospital https://orcid.org/0000-0001-7265-1556

\section{Bo Chen}

Chongqing University Cancer Hospital

\section{Bianqin Guo}

Chongqing University Cancer Hospital

\section{Xiaoyuan Deng}

Chongqing University Cancer Hospital

\section{Bin Wang}

Chongqing Medical University

\section{Xiaoyun Dou}

Chongqing Medical University

\section{Research Article}

Keywords: sevoflurane, propofol, neuroinflammation, neuroprotection, neurotoxicity, NMDA receptor

Posted Date: March 11th, 2021

DOl: https://doi.org/10.21203/rs.3.rs-280180/v1

License: (c) (i) This work is licensed under a Creative Commons Attribution 4.0 International License.

Read Full License 


\section{Abstract}

Neuroinflammation can cause cognitive deficits, and pre-existing neuroinflammation is very common in the clinic after trauma, surgery, and infection. Patients with pre-existing neuroinflammation often need further medical treatment under general anesthesia. However, it is still unknown the effects of postconditioning with general anesthetics on the pre-existing neuroinflammation. In this study, adult rats were post-treated with sevoflurane or propofol after intracerebroventricular administration of lipopolysaccharide. The effects of sevoflurane or propofol postconditioning on neuroinflammationinduced recognition memory deficit were detected. Our results found that postconditioning with sevoflurane, but not propofol reversed the selective spatial recognition memory impairment induced by neuroinflammation, and these differential effects did not appear to be associated with the similar antineuroinflammatory response of general anesthetics. However, postconditioning with propofol induced a selective long-lasting upregulation of extrasynaptic NR2B-containing NMDARs in the dorsal hippocampus, which down-regulated the CREB signaling pathway, and impaired spatial recognition memory. Additionally, the NR2B antagonists, memantine and Ro2506981, reversed this neurotoxicity induced by propofol postconditioning. Altogether, these results indicate that under the pre-existing neuroinflammation, postconditioning with sevoflurane can provide reliable neuroprotection through attenuating LPS-induced neuroinflammation, apoptosis, neuronal loss, and eventually improving spatial recognition deficit. However, although posttreatment with propofol also have the same antineuroinflammatory effects, the neurotoxicity caused by propofol postconditioning following neuroinflammation deserves to be continuously concerned.

\section{Introduction}

Sevoflurane and propofol are the most commonly used inhalation and intravenous general anesthetics in the clinic. Neuroprotective effects of sevoflurane [1-3] and propofol $[4,5]$ have been shown in the animal model of cerebral ischemia/reperfusion injury, in which they attenuated the neuroinflammation and improved cognitive function. However, conflicting results have been reported in lipopolysaccharide (LPS)induced inflammation models, one recent in vivo study showed that pretreatment with propofol could not decrease LPS-induced neuroinflammation, and have no effect on cognitive impairment in adult mice [6], but in an in vitro study, propofol attenuated LPS-induced cytokines release in cultured microglia, while isoflurane or sevoflurane could not [7]. In the clinic, neuroinflammation develops after surgery, and it is considered a major cause of postoperative cognitive dysfunction [8, 9]. Additionally, several patients in the postoperative period need further treatments when necessary including re-surgery, invasive procedures, or even sedation in the ICU, while general anesthetics are always provided in these cases, but how sevoflurane or propofol affects the pre-existing neuroinflammation and neuroinflammation-induced cognitive function is largely unknown.

Sevoflurane and propofol exert their anesthetic effects mainly through activating the gammaaminobutyric acid (GABA) receptor and inhibiting the N-methyl-D-aspartate receptor (NMDAR) [10, 11]. And the ionotropic glutamate NMDAR with different subunits plays a crucial role in regulating learning 
and memory [12]. NMDAR is a glutamate-gated ion channel, consisting of NR1, NR2 (2A-2D), and NR3 (3A, 3B) subunits. The NR1 subunit is an essential part of NMDAR, while the NR2 subunits, especially NR2A and NR2B, are mainly distributed in the adult rat hippocampus, and primarily regulate the function of the receptor [13]. Previous studies suggested that inhalation and intravenous anesthetics exhibited a differential effect on the expression of the NR2B subunit, which was accompanied by changes in cognitive function [14-17]. For example, isoflurane or sevoflurane exposure alone could improve cognitive function via an upregulation of NR2B subunit in the hippocampus $[14,16]$, while propofol exposure decreased the level of NR2B subunit in the hippocampus and induced spatial memory deficits, suggesting that upregulation of NR2B exerts the neuroprotective effect [17]. However, overexpression of the NR2B subunit is deleterious in many neurodegenerative disease models [18-20]. Indeed, NMDAR with different subcellular locations (synaptic or extrasynaptic) also contributes to the regulation of cognition by triggering the distinct signaling pathways [21, 22]. Synaptic NMDAR stimulation promotes extracellular signal-regulated kinase (ERK) activation through phosphorylation, nuclear transcription protein cAMP response element-binding (CREB) phosphorylation, and provides neuroprotective effects, whereas activation of extrasynaptic NMDAR display the opposite effect relative to synaptic receptor activation, leading to neuronal cell death. Recent studies showed that isoflurane or sevoflurane could induce developmental neurotoxicity through selectively upregulating the expression of extrasynaptic NR2B, and cause cognitive dysfunction $[23,24]$. Nevertheless, whether postconditioning with propofol or sevoflurane may regulate the expression of synaptic or extrasynaptic NMDARs subunits in the presence of preexisting neuroinflammation, and the regulation is involved in neurotoxicity or neuroprotection against LPS-induced cognitive impairment is still unknown.

In the current study, we used an established rat model of acute neuroinflammation induced by intracerebroventricular (i.c.v.) administration of LPS to investigate the effects of sevoflurane and propofol on neuroinflammation, cognitive function, and molecular alterations of NMDAR subunits in the behaviorally relevant brain region. Further, the pharmacological antagonists were used to test the role of NMDAR subunits in sevoflurane- or propofol-induced neurotoxicity or neuroprotection.

\section{Materials And Methods}

\section{Reagents}

Reagents in this study are listed in Table 1 in the Supplementary Materials.

\section{Animals}

Adult male Sprague-Dawley rats $(200 \mathrm{~g}-250 \mathrm{~g})$ from the Animal Center of Chongqing Medical University, China (permission number: SCXK 2018-0003), were housed in a 12-h light/dark cycle at $22 \pm 2{ }^{\circ} \mathrm{C}$ with food and water ad libitum. All experiments were conducted in the daytime between 8:00 and 18:00.

The animal procedures were approved by the Ethics Committee of Chongqing University Cancer Hospital and followed the Guidance Suggestions for the Care and Use of Laboratory. 
Every effort was made to minimize the number of animals used and their suffering.

\section{Drug administration}

LPS and Ro25-6981 were dissolved in the sterile artificial cerebral spinal fluid (aCSF) and administered centrally through the implanted guide cannulas as previously described $[25,26]$. Briefly, rats were anesthetized with sevoflurane and placed in the stereotaxic apparatus (RWD Life Science Co., Ltd, Shenzhen, China). For LPS administration, one stainless steel guide cannula (26-gauge) was implanted in the right lateral ventricle (-1.0 mm AP, $+1.5 \mathrm{~mm} \mathrm{ML},-3.0 \mathrm{~mm} \mathrm{DV}$; [27]) through the drilled hole in the skull. For Ro25-6981 administration, two stainless steel guide cannulas were implanted bilaterally into the dorsal hippocampus (-3.3 mm AP, +/-1.5 $1.8 \mathrm{~mm} \mathrm{ML,} \mathrm{-2.0} \mathrm{mm} \mathrm{DV;} \mathrm{[27]).} \mathrm{All} \mathrm{cannulas} \mathrm{were} \mathrm{anchored} \mathrm{to}$ the skull with steel screws and dental acrylic. A total of $10 \mu \mathrm{g}$ LPS $(5 \mu \mathrm{g} / \mu \mathrm{l})$ was administered (i.c.v.) via the implanted guide cannula at a rate of $1 \mu \mathrm{l} / \mathrm{min}$. Ro25-6981 $(0.375 \mu \mathrm{g} / \mu \mathrm{l})$ was bilaterally administered into the dorsal hippocampus via the implanted guide cannulas at a rate of $0.3 \mu \mathrm{L} / \mathrm{min}(0.5 \mu \mathrm{l}$ for each side), at a daily dose of $0.375 \mu \mathrm{g}$ for 7 consecutive days [26]. The standard histological examination was conducted to verify the placement of cannulas.

Rats who were exposed to propofol postconditioning received two consecutive intraperitoneal (i.p.) injections of propofol $(75 \mathrm{mg} / \mathrm{kg}$ ) with an interval of $30 \mathrm{~min}$. As our pilot study showed that $75 \mathrm{mg} / \mathrm{kg}$ propofol i.p. would keep the loss of righting reflex (anesthesia state) for about $30 \mathrm{~min}$. Rats who were exposed to sevoflurane postconditioning were placed in a chamber gassed with $5 \%$ sevoflurane for $2 \mathrm{~min}$ to induce anesthesia and maintained with $2 \%$ sevoflurane for $60 \mathrm{~min}$. During the whole postconditioning period, all rats inhaled pure oxygen and breathed spontaneously. Oxygen saturation was monitored using pulse oximetry and maintained above $95 \%$, and rectal temperature was monitored and maintained at $37^{\circ} \mathrm{C}$ using a heating blanket. Memantine hydrochloride $(1 \mathrm{mg} / \mathrm{ml})$ at $5 \mathrm{mg} / \mathrm{kg}$ concentration was injected (i.p.) once daily for 7 consecutive days [28].

\section{Object recognition memory tasks}

All behavioral tests were performed in a white plexiglass chamber $(50.50 .40 \mathrm{~cm})$ in a sound-attenuated room with controlled light intensity (3lux). An overhead camera was connected to a computer to record the animals' behavior. The objects used in the object recognition memory tasks varied in material (plastic, glass, metal, porcelain), shape (cylinder, cuboid, cone, irregular shapes), color (yellow, blue, green, pink), and size (15-21 cm height, 8-10 diameter). The Velcro was attached to the bottom of objects and the chamber floor to ensure that objects could not be moved by rats. Each rat was handled for 5 min followed by habituation to an empty chamber for 10 min per day for 3 consecutive days prior to the object recognition memory tasks. The time that rats spent exploring each object in all the object recognition memory tasks was analyzed offline from video records. The exploring time was measured with a stopwatch by an experimenter who was blind to the experimental design. Object exploration was only defined as directing the nose at a distance of less than $1 \mathrm{~cm}$ of the object. Turning around, sitting on the object, or touching the object with forepaws but headed in another direction were not considered exploratory behavior. 


\section{Novel object-place recognition}

This task was performed according to the standard protocol [30]. Two identical copies of an object were placed in the chamber, and rats explored them freely for 5 min (sample phase). After a 1-hour delay, one of the objects was moved to a novel place, the rat was given $3 \mathrm{~min}$ to explore (test phase). Intact rodents spend more time exploring the displaced object than the stationary one. The position of the displaced object was counterbalanced between rats. The discrimination ratio was used to represent novel objectplace preference.

Novel object-place recognition ratio $=($ Time at displaced object - Time at stationary object $) /($ Time at both objects).

\section{Novel object recognition}

Two identical copies of an object were placed in the chamber [30], and rats explored them freely for 5 min (sample phase). After $1 \mathrm{hr}$ delay, one of the objects was replaced by a novel one, and the rat was given a 3-min of exploration (test phase). Intact rodents show a significant preference for the novel object over the familiar one. The objects used as novel or old were counterbalanced between rats. The discrimination ratio was used to represent novel object preference.

Novel object recognition ratio $=($ Time at novel object - Time at old object $) /$ (Time at both objects $)$.

\section{Temporal order memory}

This test comprised 2 sample phases and 1 test phase [30]. In sample phase 1, two copies of an object were placed in the chamber, and the rat was allowed to explore freely for $5 \mathrm{~min}, 1 \mathrm{hr}$ later, two copies of a novel object were placed at the same locations in sample phase 1 , and the rat was given a 5 -min of exploration (sample phase 2). In the test phase, a third copy of the object from sample phase 1 and a third copy of the object from sample phase 2 were presented, but their locations were identical to in the sample phases, rat was given $3 \mathrm{~min}$ to explore. The positions of the objects in the test phase and the objects used in both sample phases were counterbalanced between rats. Intact rodents prefer to explore the earlier-presented object more than the recent one. The discrimination ratio was used to represent earlier-presented object preference.

Temporal order memory ratio $=($ Time at earlier-presented object - Time at recent-presented object $) /$ (Time at both objects).

\section{Tissue preparation}

Rats were anesthetized with $4 \%$ sevoflurane for 3 min. For enzyme-linked immunosorbent assay (ELISA) and western blot, the perirhinal cortex $(\mathrm{PRH})$, the dorsal and ventral hippocampi were separately dissociated and stored at $-80^{\circ} \mathrm{C}$. 
For immunohistochemistry, Nissl staining, and immunofluorescence, rats were transcardially perfused with $0.9 \%$ saline followed by $4 \%$ paraformaldehyde (PFA) solution.

The brains were removed and postfixed in 4\% PFA solution for $24 \mathrm{~h}$. The right halves of the brains were embedded in paraffin and cut into $4 \mu \mathrm{m}$ thick coronal sections for immunohistochemistry and Nissl staining. The left halves of the brains were cut into $50 \mu \mathrm{m}$ thick sections using a vibratome (VT1000, Leica) for immunofluorescence.

\section{Immunohistochemistry}

Immunohistochemistry was performed as previously described [31]. Every fifth paraffin sections containing dHPC (-2.5 -4 AP) were deparaffinized in xylene and rehydrated in a serial of graded ethanol, which was followed by antigen retrieval with $0.1 \mathrm{M}$ sodium citrate buffer $(\mathrm{pH} 6.0)$ in a microwave oven for 20 min. After blocking with $0.3 \%$ hydrogen peroxide and $5 \%$ normal goat serum, sections were incubated with the primary antibody against cleaved caspase-3 $(1: 100)$ overnight at $4^{\circ} \mathrm{C}$, sections then were incubated with the second goat anti-rabbit $\operatorname{lgG}$ antibody at $37^{\circ} \mathrm{C}$ for $30 \mathrm{~min}$. Finally, sections were stained with DAB solution. Hematoxylin was used for nuclear staining. Negative sections were incubated with PBS instead of primary antibody. The number of cleaved caspase-3 positive neurons within the CA1 region of dHPC was counted in images from five microscopic areas under 200x magnification by an investigator who was blinded to the experiments. All images were analyzed using Image $\mathrm{J}$ software.

\section{Nissl staining}

Nissl staining was performed as previously described [32]. The deparaffinized sections containing dHPC were incubated with $1 \%$ cresyl violet for $30 \mathrm{~min}$ at $37^{\circ} \mathrm{C}$, then dehydrated in a serial of graded ethanol, cleared and coverslipped. The number of neurons was calculated using the method mentioned in the immunohistochemical experiment.

\section{Immunofluorescence}

Immunofluorescence was performed as previously described [31]. Sections were blocked with $5 \%$ normal goat serum and $0.4 \%$ Triton X-100 in PBS for $1 \mathrm{~h}$ at $37^{\circ} \mathrm{C}$. Then sections were incubated with the primary antibodies against iba- $1(1: 1000)$ or NR2B $(1: 1000)$ for $48 \mathrm{hr}$ at $4^{\circ} \mathrm{C}$. After 3 times washing, sections were incubated with the second goat anti-rabbit IgG Alexa Fluor 488 (1:500) or goat anti-rabbit IgG Alexa Fluor 647 (1:500) for $2 \mathrm{hr}$ in the dark at $37^{\circ} \mathrm{C}$ and counterstained with $0.5 \%$ DAPI for $3 \mathrm{~min}$ at room temperature. The number of iba-1 positive cells in the dHPC was calculated from six sections using a confocal microscope (Leica TCS SP8, Wetzlar, Germany) under 200x magnification (NA 0.85).

\section{ELISA}

The bilateral dHPC were homogenized in RIPA buffer and centrifuged at $12000 \mathrm{~g}$ for $10 \mathrm{~min}$ at $4^{\circ} \mathrm{C}$. The supernatant was collected and the protein concentration was determined using BCA assay kits. The protein concentration in all samples was adjusted to $4 \mathrm{mg} / \mathrm{ml}$. ELISA was performed following the manufacturer's instructions, and absorbance was quickly read using a 96-well plate reader at a detection 
wavelength of $450 \mathrm{~nm}$ as previously described [33]. The concentrations of TNF- $\mathrm{a}$ and IL-1 $\beta$ were presented as $\mathrm{pg} / \mathrm{mg}$ protein. In our preliminary experiment, contents of TNF- $\alpha$ and IL- $1 \beta$ in the left and right dHPC were measured separately, and we did not find any significant differences in LPS-induced cytokine concentrations between the contralateral and the ipsilateral brain tissues of dHPC. So we used the whole bilateral dHPC for ELISA and subsequent western blot.

\section{Subcellular fractionation}

Subcellular fractionation of dHPC was performed using an adapted protocol [34-36] with minor modification. Tissues were homogenized by sonication in buffer $A$ containing $0.32 \mathrm{M}$ sucrose, and $10 \mathrm{mM}$ HEPES (pH7.4). The homogenate $(\mathrm{H})$ was centrifuged at $1000 \mathrm{~g}$ for $10 \mathrm{~min}$ to obtain the pellet (P1) containing nuclei and cellular debris, and the supernatant (S1) was centrifuged at $10,000 \mathrm{~g}$ for $15 \mathrm{~min}$ to obtain the crude synaptosomal membrane fraction (P2) and the supernatant (S2). The S2 was centrifuged at $165,000 \mathrm{~g}$ for $2 \mathrm{hr}$ to obtain a cytosolic fraction (S3) and a microsome-enriched fraction (P3). The crude synaptosomal membrane pellet (P2) was separated by $0.85 / 1.0 / 1.2 \mathrm{M}$ sucrose density gradient centrifugation (825,000 $\mathrm{g}$ for $2 \mathrm{hr}$ ). The synaptosomes were obtained from the 1.0/1.2 $\mathrm{M}$ sucrose interface, and synaptosomal pellets were resuspended in buffer B containing $0.5 \%$ Triton $X-100,20 \mathrm{mM}$ HEPES (pH7.2). Then the suspension was incubated for $30 \mathrm{~min}$ with gentle rotation and centrifuged at $320,000 \mathrm{~g}$ for $20 \mathrm{~min}$. Based on the fact that PSDs and synaptic junctions are insoluble in Triton X-100 $[37,38]$. The supernatant, TxS (Triton X-100 soluble), is defined as the non-PSDs membrane-enriched protein or extra-synaptic fraction. The pellet, TxP (Triton X-100 insoluble), is defined as the PSDs membrane-enriched protein or synaptic fraction.

Nuclear fractionation was performed as previously described [39]. Briefly, P1 were resuspended in buffer C containing 10mM HEPES (pH7.4), $10 \mathrm{mM} \mathrm{KCl}, 10 \mathrm{mM}$ EDTA, $1.5 \mathrm{mM} \mathrm{MgCl}_{2}, 0.2 \% \mathrm{BSA}, 1 \mathrm{mM}$ DTT, and $0.4 \% \mathrm{NP} 40$. After incubation with gentle rotation for $15 \mathrm{~min}$, the suspension was centrifuged at $15,000 \mathrm{~g}$ for $10 \mathrm{~min}$ to obtain the pellets which are defined as crude nucleus fraction. The crude nucleus fraction was then resuspended in buffer $\mathrm{D}$ containing $20 \mathrm{mM}$ HEPES (pH7.4), 400mM NaCl, $1 \mathrm{mM}$ EDTA, $10 \%$ Glycerol, and $1 \mathrm{mM}$ DTT. The suspension was incubated for $2 \mathrm{hr}$ with gentle rotation followed by centrifugation (15,000 $\mathrm{g}$ for $10 \mathrm{~min}$ ) to obtain the supernatant (nuclear fraction). All procedures mentioned above were performed at $4{ }^{\circ} \mathrm{C}$ and all buffers contained protease and phosphatase inhibitor cocktail. Protein concentrations in all fractions were determined by BCA protein assay and adjusted for western blot.

\section{Western blot}

The ventral hippocampal and PRH tissues were lysed in ice-cold RIPA buffer containing protease inhibitor cocktail and centrifuged at $12000 \mathrm{~g}$ for $10 \mathrm{~min}$ at $4^{\circ} \mathrm{C}$. The supernatants were collected and protein concentration was determined using BCA assay kits. Western blot was performed as described previously [40]. In brief, each sample containing $10 \mu \mathrm{g}$ protein was separated on an $8 \%$ sodium dodecyl sulfate gel by electrophoresis and transferred to a $0.45 \mu \mathrm{m}$ polyvinylidene fluoride membrane. Non-specific protein binding was blocked with $5 \%$ milk in TBST for $60 \mathrm{~min}$ at room temperature. The membranes were 
incubated with primary antibodies of NR2A (1:1000), NR2B (1:1000), NR1 (1:1000), NR3A (1:1000), NR3B (1:1000), GluR1 (1:1000), GluR2 (1:1000), PSD-95 (1:1000), pERK1/2 (1:1000), ERK1/2 (1:1000), $\operatorname{pCREB}(1: 1000), \operatorname{CREB}(1: 1000)$, Calnexin (1:5000), Synaptophysin (1:10000) and $\beta$-actin (1:10000) overnight at $4^{\circ} \mathrm{C}$, and then incubated with goat anti-rabbit IgG-HRP (1:5000) or goat anti-mouse IgG-HRP (1:5000) for $1 \mathrm{hr}$ at room temperature. The protein bands were detected by enhanced chemiluminescence reagents and photographed. The results were analyzed using an image acquisition system (Fusion FX7, Vibert Lourmat, France). For stripping and reprobing, membranes were washed in TBST and incubated in stripping buffer for $60 \mathrm{~min}$ at room temperature.

\section{Statistical analysis}

Statistical analysis was performed with SPSS (version 22. IBM, New York, NY, USA), and data were presented as mean \pm SEM. A three-way ANOVA was used to analyze the data from ELISA. For the object recognition memory tasks, both the total object exploring time and the discrimination ratio were analyzed with a two-way ANOVA followed by Turkey post hoc analysis; and a one-sample t-test was used to compare the ratio with zero (chance level). All other data were analyzed using a two-way ANOVA followed by Turkey post hoc analysis. A significant level of $p<0.05$ was used.

\section{Results}

\section{Postconditioning with propofol or sevoflurane exerts a differential effect on novel object-place recognition impairment induced by neuroinflammation}

Acute neuroinflammation mimics several pathological conditions during the perioperative period in the clinic $[8,9]$. To investigate the effect of postconditioning with general anesthetics on neuroinflammationinduced recognition impairment, rats were subjected to i.c.v. administration of LPS $(10 \mu \mathrm{g})$, followed by postconditioning with propofol or sevoflurane. Rats who received the equal volume of aCSF instead of LPS served as Control, and intralipid was used for the vehicle solution of propofol. On day 7 after the postconditioning, all rats were tested for a serial of object recognition memory experiments (Fig. 1a).

Control rats exhibited positive values of discrimination ratio in the novel object-place recognition test, indicating that rats had intact memory for object place. Compared with control rats, LPS-injected rats exhibited impaired novel object-place recognition memory as shown by a significantly decreased discrimination ratio. Surprisingly, only postconditioning with sevoflurane, but not propofol, reversed this impairment. Sevoflurane or propofol exposure in control rats did not have a significant effect on the discrimination ratio (Fig. 1b). There were no differences among groups in the total time of object exploration during the sample or test phase, indicating that the predisposition to explore objects was not affected (Supplementary Fig. 1a).

Next, rats were tested in novel object recognition memory and temporal order memory. In the novel object recognition memory test, rats in all groups showed a significantly higher preference for the novel object, suggesting that all rats had intact memory for object identity. And there was no significant difference in 
the discrimination ratio among groups (Fig. 1c). The total time of object exploration during the sample or test phase was not different among groups (Supplementary Fig. 1b). In the temporal order memory test, rats in all groups exhibited a significantly higher preference for the earlier-presented object as shown by the similar levels of discrimination ratio (Fig. 1d). The differences in the total time of object exploration during sample1, sample2, or test phase were not observed among groups (Supplementary Fig. 1c). These results showed that acute LPS injection selectively caused spatial recognition memory impairment, and postconditioning with sevoflurane, but not propofol, provided protection against this impairment.

\section{Postconditioning with propofol or sevoflurane exerts a similar anti-inflammatory effect}

Next, we examined whether the differential effect of postconditioning with sevoflurane or propofol on LPS-induced novel object-place recognition impairment was due to their different anti-inflammatory effect by measuring the time course of expression of pro-inflammatory cytokines in the dHPC, which is highly associated with spatial recognition memory. After LPS injection, pro-inflammatory cytokine TNF-a increased and peaked at $6 \mathrm{hr}$, and returned to the basal level on day 1 . Both postconditioning with propofol and sevoflurane slightly decreased the level of TNF-a. Propofol or sevoflurane exposure did not produce a significant change of TNF- $\alpha$ in control rats (Fig. 2a). Another pro-inflammatory cytokine IL-1 $\beta$ increased markedly from $6 \mathrm{hr}$ and remained significantly high till $24 \mathrm{hr}$ after LPS injection. Posttreatment with propofol reversed the lasting IL-1 $\beta$ upregulation at $6 \mathrm{hr}$ and $24 \mathrm{hr}$, while the similar reversal effect was seen with sevoflurane at $6 \mathrm{hr}$ (Fig. 2b).

Since excessive synthesis and release of pro-inflammatory cytokines is triggered by activated microglia [41], we next examined the microglia activation by measuring the total number of iba1 (a specific microglia marker) positive cells in the dHPC. Compared with control rats, the number of microglia was significantly increased both on day 3 and day 7 in LPS-injected rats. Posttreatment with propofol or sevoflurane dramatically relieved LPS-induced increased number of microglia. Propofol or sevoflurane exposure did not affect the total number of microglia in control rats (Fig. 2c, d). Taken together, these results suggested that postconditioning with propofol or sevoflurane exerted a similar anti-inflammatory effect after LPS injection.

\section{Postconditioning with propofol or sevoflurane exerts differential effects on apoptosis and neuronal loss}

Given that neuronal apoptosis has been proved as a notable event of LPS-induced neuroinflammation [42-44], and postconditioning with sevoflurane or propofol can provide neuroprotection through inhibition of neuronal apoptosis in the model of cerebral ischemia/reperfusion injury $[2,4]$. We then sought to examine whether the differential effect of postconditioning with sevoflurane or propofol on LPS-induced spatial recognition deficit resulted from their different role in mediating apoptosis. Using immunohistochemistry staining, we detected the number of cleaved caspase-3 (a marker of apoptosis) positive neurons in the CA1 region of dHPC. Undoubtedly, the number of apoptotic neurons was 
dramatically increased on day 1 and day 7 after LPS injection, and this pro-apoptotic effect was partially alleviated by postconditioning with sevoflurane or propofol on day 1 . However, a significant increase in the number of cleaved caspase-3 positive neurons was still observed on day 7 after propofol postconditioning, whereas posttreatment with sevoflurane almost completely restored the increased number of apoptotic neurons to normal (Fig. 3a, b). The notable difference in anti-apoptotic effect induced by propofol or sevoflurane postconditioning prompted us to investigate the number of pyramidal neurons in the dorsal hippocampal CA1 region on day 7. We found that LPS alone induced a significant decrease in the number of Nissl stained pyramidal neurons, and postconditioning with sevoflurane, but not propofol reversed this neuronal loss (Fig. 3c, d).

\section{Postconditioning with propofol or sevoflurane exerts a differential effect on the expression pattern of NMDA receptor subunits}

Propofol and sevoflurane can differently mediate the expression of NMDARs in the absence of acute neuroinflammation $[16,17]$, and our observation that postconditioning with propofol or sevoflurane exhibited a similar anti-inflammatory effect, but only sevoflurane, not propofol, induced a robust and persistent anti-apoptotic effect and reversed the LPS-induced spatial recognition memory impairment let us speculate that besides the neuroprotective effect provided by the anti-inflammation response, changes of NMDARs may contribute to the neuronal damage and spatial recognition memory deficit after posttreatment with propofol. To test this possibility, we detected the expression of NMDAR subunits in the dHPC, and we discovered that LPS alone did not affect the amount of NR2B subunit in total dHPC protein homogenates either on day 1 or day 7 compared with control-vehicle rats. However, postconditioning with propofol induced a long-lasting, almost 1.5-fold increase in NR2B subunit expression both on day 1 and day 7. In contrast, postconditioning with sevoflurane induced a slight increase in NR2B expression on day 1 , while no change was observed on day 7. Interestingly, neither propofol nor sevoflurane changed the NR2B subunit expression in control rats (Fig. 4a, b). This modulation of NR2B subunit expression induced by propofol postconditioning could also be seen by immunofluorescence (Fig. 4e). Meanwhile, no changes were observed in the expression of NR2A or NR1 indicating that postconditioning with propofol specifically modulated the NR2B expression in the presence of pre-existing neuroinflammation (Fig. 4a, C, d). We also detected other ionotropic glutamate receptor subunits, a-amino-3-hydroxy-5-methyl-4isoxazole propionic acid receptors (AMPARs) subunits, and other NR2 subunits for their responses to general anesthetics postconditioning after LPS injection. In normal adult rats, AMPARs subunits, both GluR1 and GluR2, are expressed at high levels in the forebrain including the hippocampus, while GluR3, NR2C, and NR2D are expressed at relatively low levels $[45,46]$. We discovered that the total levels of GluR1, GluR2, GluR3, NR2C, and NR2D were not altered in the dHPC of rats among all groups (Supplementary Fig. 2a-f). Moreover, the specific upregulation of NR2B induced by propofol postconditioning was not observed in other brain regions including the ventral hippocampus(VHPC) or PRH (Supplementary Fig. 2g-i).

NR2A-containing NMDARs are mainly localized at postsynaptic sites via binding with PSD-95 (a postsynaptic scaffolding protein), and synaptic NMDARs activation mediates physiologic effect and 
promotes cell survival. NMDARs also exist at the extrasynaptic site, in which the NR2B subunit is relatively enriched, and overactivation of extrasynaptic NR2B leads to neuronal death $[47,48]$. Thus, we further investigated the changes of NMDAR subunits both at synaptic and extrasynaptic sites in the dHPC on day 7 after general anesthetics postconditioning. Firstly, biochemical fractionation was used to separate synaptic (TxP) and extrasynaptic (TxS) fractions, and the effectiveness of the fractionation was confirmed by synaptophysin, PSD95 (Fig. 4f), and calnexin (Supplementary Fig. 3) as markers for the subcellular compartments as described previously [39,49]. No significant differences in synaptic or extrasynaptic NR2B levels were observed after LPS injection, however, postconditioning with propofol significantly increased NR2B levels in extrasynaptic but not synaptic fractions. NR2B levels both in synaptic and extrasynaptic fractions were not changed after sevoflurane postconditioning. Meanwhile, the levels of NR2A and NR1 either in synaptic or extrasynaptic remained unaffected (Fig. 4g-j).

Overstimulation of extrasynaptic NR2B-containing NMDARs causes inactivation of ERK1/2 and dephosphorylation of nuclear CREB and ultimately leads to neuronal death $[21,22,47,48]$. Therefore, we determined the involvement of the CREB signaling pathway in excessive activation of extrasynaptic NR2B induced by propofol postconditioning. The phosphorylation level of ERK1/2 in dHPC protein homogenate was significantly decreased in rats who were post-treated with propofol, whereas the total protein level was unchanged (Fig. 4k, i). Similarly, the phosphorylation level of CREB in dHPC protein nuclear fraction was also significantly decreased when rats were post-treated with propofol, without a corresponding change in total CREB (Fig. 4k, j). In contrast, the phosphorylation levels of ERK1/2 and CREB were unchanged in rats from other groups. Altogether, these results suggested that postconditioning with propofol selectively induced a long-lasting increase of extrasynaptic NR2B expression in the dHPC, which was accompanied by inactivation of the downstream CREB signaling pathway.

\section{Persistent upregulation of extrasynaptic NR2B subunits may contribute to the neurotoxicity effect of postconditioning with propofol on neuronal apoptosis and spatial cognitive impairment following neuroinflammation}

Based on all the above results obtained, we next used pharmacological antagonists to confirm the effect of persistent upregulation of extrasynaptic NR2B on neurotoxicity induced by propofol postconditioning after LPS injection. The non-competitive antagonist memantine which selectively inhibits the extrasynaptic NMDARs at a low dose was administered i.p. for 7 consecutive days after postconditioning with propofol as previously described $[28,50]$. An equal volume of saline was administered in sham rats (Fig. 5a). On day 7 after postconditioning, all rats were tested for the novel object-place recognition memory followed by molecular biological detection (Fig. 5a). Memantine treatment remarkably reversed the elevated levels of NR2B induced by propofol postconditioning both in total dHPC protein homogenate and extrasynaptic fraction, whereas it did not affect the expression of NR2A and NR1 subunits in each subcellular fraction (Fig. 5b-e). In addition, memantine treatment reversed the CREB signaling deficit as shown by significantly increased phosphorylation levels of ERK1/2 and CREB (Fig. 5f-h). Propofol postconditioning-induced apoptosis and neuronal loss were also reversed by memantine (Fig. 5i-k). Finally, memantine rescued the spatial recognition memory impairment induced by propofol 
postconditioning following neuroinflammation, without altering the predisposition to explore objects (Fig. 5I, m). Another NR2B antagonist, Ro25-6981 (IC50: 9nM for NR2B/NR1, 52 $\mu$ M for NR2A/NR1) [51], was locally administered in the dHPC for 7 consecutive days after postconditioning with propofol. Similarly, the elevated levels of NR2B in total dHPC homogenate and extrasynaptic fraction, the decreased levels of phosphorylation ERK1/2 and nuclear CREB, and the increases in apoptosis and neuronal loss induced by propofol postconditioning were reversed by Ro25-6981 treatment (Fig. 5b-k). Additionally, spatial recognition memory impairment was improved in Ro25-6981-treated rats compared with sham rats (Fig. $5 \mathrm{I}, \mathrm{m}$ ). Taken together, these results indicated that persistent upregulation of extrasynaptic NR2B subunit in the dHPC may contribute to the neurotoxicity effect of postconditioning with propofol on spatial recognition memory impairment in the presence of pre-existing neuroinflammation.

\section{Discussion}

Surgery or trauma can cause systemic inflammation, and damage the brain-blood barrier, eventually induce neuroinflammation [52], which has been proved to be highly associated with postoperative cognitive dysfunction $[8,9,53]$. Intracerebroventricular injection of LPS can mimic neuroinflammation induced by surgery or trauma in animal studies $[25,54]$. In the present study, we discovered that LPSinduced neuroinflammation increased apoptosis and neuronal loss in the $\mathrm{dHPC}$, and ultimately lead to a selective spatial recognition memory impairment. This finding is consistent with previous studies which demonstrated that spatial memory is more vulnerable than non-spatial memory to LPS-induced damage $[25,54,55]$. In fact, the processing of spatial recognition memory is highly dependent on the dHPC [30, 56], while non-spatial recognition memory judgments for individual items or relative recency of items primarily depend on other brain regions, including $\operatorname{mPFC}$ and $\mathrm{PRH}[57,58]$. We further found that postconditioning with propofol or sevoflurane exerted a similar anti-neuroinflammatory effect via inhibiting microglia activation and the release of proinflammatory cytokines. However, postconditioning with sevoflurane but not propofol attenuated the apoptosis and neuronal loss and completely rescued the spatial cognitive impairment induced by neuroinflammation. Finally, we discovered that the persistent upregulation of extrasynaptic NR2B and the downstream CREB signaling pathway were involved in the neurotoxicity effect of postconditioning with propofol in the presence of pre-existing neuroinflammation.

An interesting finding of the present study is that only posttreatment with sevoflurane, but not propofol reversed the spatial recognition memory impairment after LPS injection. This is an intriguing observation because both inhalation and intravenous anesthetics are generally thought to have a neuroprotective effect in various experimental models including myocardial infarction [59], cerebral ischemia/reperfusion injury [1-5], and hemorrhage shock [60]. But recently, differential effects of sevoflurane and propofol on anxiety-like behavior in formalin-induced pain were reported in an animal study [61]. A clinical study also showed that sevoflurane-based anesthesia for on-pump cardiac surgery was associated with better shortterm postoperative cognitive performance than propofol [62], however, the mechanism underlying this differential effects of sevoflurane and propofol on postoperative cognitive function has been unknown. Here, we found that postconditioning with sevoflurane persistently alleviated apoptosis and neuronal loss 
in the dHPC after LPS injection, whereas the anti-apoptotic effect of propofol postconditioning was only observed on one day after LPS injection, and increased apoptosis and neuronal loss still existed at a later time. One possible explanation for these results is that the anti-inflammatory effect of propofol postconditioning was more prominent than neurotoxicity in an early stage of LPS-induced acute neuroinflammation, while the neurotoxic effect was long-lasting and may account for the neuronal loss in the dHPC.

NR2B subunit is crucial to vital brain functions including synaptic plasticity, gene transcription, neuronal survival, and cognitive function [12, 63, 64]. After maturation, NR2B-containing NMDARs are mainly distributed at the extrasynaptic site, and excessive activation of extrasynaptic NR2B-containing receptors leads to neuronal death via activating downstream signaling pathways [20-24]. We found a selective increase of extrasynaptic NR2B-containing receptors after propofol postconditioning. In contrast, NR2B at the synaptic site was insensitive to propofol postconditioning. Moreover, other NMDAR subunits or AMPAR subunits were not modulated by propofol postconditioning. Meanwhile, we did not find LPS alone could alter NR2B expression, indicating the effect of propofol postconditioning on NR2B expression was exerted directly by itself, but not secondary to the anti-inflammation. Phosphorylation of ERK $1 / 2$ and CREB, as the downstream signaling molecules of synaptic NMDARs, play an important role in modulating the transcription of genes that promote spine formation, synaptic plasticity, neuronal survival, learning and memory [20]. In the current study, posttreatment with propofol reduced the levels of total ERK1/2 and nuclear CREB phosphorylation, which is associated with up-regulation of extrasynaptic NR2B-containing NMDARs. In addition, the increases in total and extrasynaptic NR2B-containing receptors expression and neuronal loss, deficits in downstream CREB signaling pathway, along with associated spatial recognition memory impairment induced by propofol postconditioning were reversed by systematical treatment with low dose memantine, previously shown to selectively block extrasynaptic NMDAR current while preserving synaptic NMDAR current [18]. Treatment with a low dose of memantine alone did not affect behavioral performance in distinct memory tasks [65], however, memantine at a 30-fold higher dose, which should block both synaptic and extrasynaptic NMDAR current, worsened the neurodegeneration and impaired cognitive function [18, 65]. Meanwhile, another NR2B antagonist Ro25-6981 was used to confirm the effect of persistent upregulation of extrasynaptic NR2B on neurotoxicity induced by propofol postconditioning after LPS injection. Ro25-6981 is commonly applied by local brain region injection or intraperitoneal injection in animal studies $[66,67]$. And several studies showed that antagonism of NR2B by Ro25-6981 could inhibit its up-regulation $[68,69]$. Here, consecutive administrations of Ro25-6981 at a very low dose $(0.375 \mu \mathrm{g})$ inhibited up-regulation of total and extrasynaptic NR2B subunit in the dHPC, reversed down-regulation of ERK1/2 and CREB phosphorylation, excessive apoptosis, neuronal loss, and improved the cognitive deficit after propofol postconditioning. We did not examine the effects of memantine and Ro25-6981 on normal object-place recognition memory, but it is known that memantine or Ro25-6981 produces its pharmacological effect in a dose-dependent manner [66, 70], and it was confirmed that Ro25-6981 with $0.375 \mu \mathrm{g}$ did not impair the normal object recognition memory, but only eliminated the changes of recognition memory induced by NR2B subunit alteration, while a high dose of

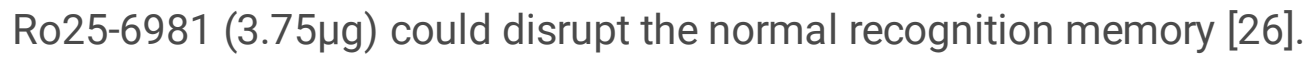


Many conflicting observations regarding the effects of general anesthetics on NR2B expression and cognitive function were reported previously. For instance, isoflurane up-regulated extrasynaptic NR2B in the hippocampus in elderly rats and impaired cognitive function [24]; and isoflurane or sevoflurane upregulated NR2B in the hippocampus, facilitated LTP in CA1 area, and improved cognition in adult mice $[14,16]$. Sevoflurane induced NR1 and NR2B up-regulation, NR2A down-regulation in the PFC, and caused cognitive deficits in adolescent rats [71], while propofol down-regulated NR1, NR2B, NR2A, and PSD-95 in the PFC of developing rat brain, and caused long-term cognitive dysfunction $[17,72]$. The possible reasons for this discrepancy might be different ages, species of animals, and strategies of drug treatment. Moreover, the above studies were more concerned with the effects of general anesthetics exposure alone on NR2B expression and cognitive function. However, when LPS-induced neuroinflammation has already existed, general anesthetics may exert different effects on NR2B expression and cognitive function. It is still unknown why postconditioning with sevoflurane or propofol has different modulation on NR2B expression in the present study. One possibility is that although sevoflurane and propofol exert their anesthetic effect through GABA receptor and NMDA receptor, NR2B subunit is more sensitive to propofol than sevoflurane [73,74]. Besides, many posttranslational mechanisms including protein-protein interaction, phosphorylation, palmitoylation, ubiquitination, and proteolysis contribute to regulating the specific synaptic and extrasynaptic NMDARs distribution [75], but to our knowledge, only a few studies demonstrated that general anesthetics ur-regulated the expression of serine/threonine cyclin-dependent kinase 5 (Cdk5) [76,77], which is implicated in regulating the surface expression of NMDARs by NR2B phosphorylation at tyrosine1472 [78]. Therefore, further study should be conducted to elucidate the exact mechanism of the selective increase of extrasynaptic NR2B-containing NMDARs induced by propofol postconditioning after LPS injection.

In summary, under the pre-existing neuroinflammation, postconditioning with sevoflurane can provide reliable neuroprotection through attenuating LPS-induced neuroinflammation, apoptosis, neuronal loss, and improving spatial recognition deficit. However, posttreatment with propofol leads to a selective longlasting upregulation of extrasynaptic NR2B-containing NMDARs in the dHPC, which down-regulates the CREB signaling pathway and impair the spatial recognition memory. The differential effects of postconditioning with sevoflurane or propofol suggest that sevoflurane seems to be more appropriate than propofol in preserving cognitive function.

\section{Declarations}

\section{Authors' contributions Conceptualization}

Bo Chen: Conceptualization, Methodology, Formal analysis, Investigation, Writing - original draft, Funding acquisition. Bianqin Guo: Methodology, Investigation. Xiaoyuan Deng: Formal analysis, Investigation, Writing - original draft. Bin Wang: Methodology, Investigation, Writing - review \& editing. Xiaoyun Dou: 
Investigation. Hongliang Liu: Conceptualization, Methodology, Writing - original draft, Writing - review \& editing, Funding acquisition.

\section{Funding}

This study was supported by grants from the National Science Foundation Project of Chongqing (cstc2017jcyjBX0043 and cstc2019jcyj-msxmX0608).

\section{Data Availability}

The data used during the present study are available from the corresponding author upon reasonable request.

\section{Compliance with Ethical Standards}

This study was conducted "according to the recommendations in the guide for the care and use of laboratory animals of the National Institutes of Health $(\mathrm{NIH})$ and the approval of the committee on the Ethics of Animal Experiments of the Chongqing University Cancer Hospital (Chongqing, P.R. China: Approval No. SCXK 2018-0003).

\section{Conflict of Interest}

The authors have no declarations of interest to declare that are relevant to the content of this article.

\section{Consent to Participate}

Not applicable.

\section{Consent for Publication}

All authors consent to the publication of this manuscript.

\section{Acknowledgments}

We wish to thank Lixue Chen, Guangcheng Qing and Dunke Zhang for excellent technical assistance.

\section{References}

1. Kitano H, Kirsch JR, Hurn PD, Murphy SJ (2007) Inhalational anaesthetics as neuroprotectants or chemical preconditioning agents in ischemic brain. J Cereb Blood Flow Metab 27(6):1108-1128. https://doi.org/10.1038/sj.jcbfm.9600410

2. Wang J-K, Yu L-N, Zhang F-J, Yang M-J, Yu J, Yan M, et al (2010) Postconditioning with sevoflurane protects against focal cerebral ischemia and reperfusion injury via PI3K/Akt pathway. Brain Res 1357:142-151. https://doi.org/10.1016/j.brainres.2010.08.009 
3. Hwang J-W, Jeon Y-T, Lim Y-J, Park H-P (2017) Sevoflurane postconditioning-induced antiinflammation via inhibition of the toll-like receptor-4/nuclear factor kappa B pathway contributes to neuroprotection against transient global cerebral ischemia in rats. Int J Mol Sci. 18(11):2347. https://doi.org/10.3390/ijms18112347

4. Wang H, Wang G, Yu Y, Wang Y (2009) The role of phosphoinositide-3-kinase/Akt pathway in propofol-induced postconditioning against focal cerebral ischemia-reperfusion injury in rats. Brain Res 1297:177-184. https://doi.org/10.1016/j.brainres.2009.08.054

5. Liang C, Cang J, Wang H, Xue Z (2013) Propofol attenuates cerebral ischemia/reperfusion injury partially sing heme oxygenase-1. J Neurosurg Anesthesiol 25(3):311-316. https://doi.org/10.1097/ANA.0b013e31828c6af5

6. Huang C, Ng OT-W, Chu JM-T, Irwin MG, Hu X, Zhu S, et al (2019) Differential effects of propofol and dexmedetomidine on neuroinflammation induced by systemic endotoxin lipopolysaccharides in adult mice. Neurosci Lett 707:134309. https://doi.org/10.1016/j.neulet.2019.134309

7. Ye X, Lian Q, Eckenhoff MF, Eckenhoff RG, Pan JZ (2013) Differential general anesthetic effects on microglial cyktokine expression. PLos One. 8(1): e52887. https://doi.org/10.1371/journal.pone.0052887

8. Shaw K, Commins S, O'Mara SM (2001) Lipopolysaccharide causes deficits in spatial learning in the water maze but not in BDNF expression in the rat dentate gyrus. Behav Brain Res 124(1):47-54. https://doi.org/10.1016/s0166-4328(01)00232-7

9. Sparkman NL, Kohman RA, Scott VJ, Boehm GW (2005) Bacterial endotoxin-induced behavioral alterations in two variations of the Morris water maze. Physiol Behav 86(1-2):244-251. https://doi.org/10.1016/j.physbeh.2005.07.016

10. Irifune M, Takarada T, Shimizu Y, Endo C, Katayama S, Dohi T, et al (2003) Propofol-induced anaesthesia in mice is mediated by gamma-aminobutyric acid-A and excitatory amino acid receptors. Anesth Analg 97(2):424-429. https://doi.org/10.1213/01.ane.0000059742.62646.40

11. Kelly EW, Solt K, Raines DE (2007) Volatile aromatic anaesthetics variably impact gammaaminobutyric acid type A receptor function. Anesth Analg 105(5):1287-1292. https://doi.org/10.1213/01.ane.0000282829.21797.97

12. Newcomer JW, Krystal JH (2001) NMDA receptor regulation of memory and behavior in humans. Hippocampus 11(5):529-542. https://doi.org/10.1002/hipo.1069

13. Furukawa H, Singh SK, Mancusso R, Gouaux E (2005) Subunit arrangement and function in NMDA receptor. Nature 438(7065):185-192. https://doi.org/10.1038/nature04089

14. Rammes G, Starker LK, Haseneder R, Berkmann J, Plack Alexandra, Zieglgänsberger W (2009) Isoflurane anaesthesia reversibly improve cognitive function and long-term potentiation (LTP) via an up-regulation in NMDA receptor 2B subunit expression. Neuropharmacology 56(3):626-636. https://doi.org/10.1016/j.neuropharm.2008.11.002

15. Monaco SA, Gulchina Y, Gao W-J (2015) NR2B subunit in the prefrontal cortex: A double-edged sword for working memory function and psychiatric disorders. Neurosci Biobehav Rev 56:127-138. 
https://doi.org/10.1016/j.neubiorev.2015.06.022

16. Liu J, Zhang X, Zhang W, Gu G, Wang P (2015) Effects of sevoflurane on young male adult C57BL/6 mice spatial cognition. PLoS One 10(8):e0134217. https://doi.org/10.1371/journal.pone.0134217

17. Wang Y, Han S, Han R, Su Y, Li J (2017) Propofol-induced downregulation of NR2B membrane translacation in hippocampus and spatial memory deficits of neonatal mice. Brain Behav 7(7):e00734. https://doi.org/10.1002/brb3.734

18. Okamoto S, Pouladi MA, Talantova M, Yao D, Xia P, Ehrnhoefer DE, et al (2009) Balance between synaptic versus extrasynaptic NMDA receptor activity influences inclusions and neurotoxicity of mutant huntingtin. Nat Med 15(12):1407-1413. https://doi.org/10.1038/nm.2056

19. Li S, Jin M, Koeglsperger T, Shepardson NE, Shankar GM, Selkoe DJ (2011) Soluble Aß oligomers inhibit long-term potentiation through a mechanism involving excessive activation of extrasynaptic NR2B-containing NMDA receptors. J Neurosci 31(18):6627-6638. https://doi.org/10.1523/JNEUROSCI.0203-11.2011

20. Hardingham GE, Bading $\mathrm{H}$ (2010) Synaptic versus extrasynaptic NMDA receptor signalling: implications for neurodegenerative disorders. Nat Rev Neurosci 11(10):682-696. https://doi.org/10.1038/nrn.2911

21. Hardingham GE, Fukunaga Y, Bading H (2002) Extrasynaptic NMDARs oppose synaptic NMDARs by triggering CREB shut-off and cell death pathways. Nat Neurosci 5(5):405-414. https://doi.org/10.1038/nn835

22. Gouix E, Léveillé F, Nicole O, Melon C, Had-Aissouni L, Buisson A (2009) Reverse glial glutamate uptake triggers neuronal cell death through extrasynaptic NMDA receptor activation. Mol Cell Neurosci 40(4):463-473. https://doi.org/10.1016/j.mcn.2009.01.002

23. Wang W-Y, Jia L-J, Luo Y, Zhang H-H, Cai F, Mao H, et al (2016) Location- and subunit-specific NMDA receptors determine the developmental sevoflurane neurotoxicity through ERK1/2 signaling. Mol Neurobiol 53(1):216-230. https://doi.org/10.1007/s12035-014-9005-1

24. Li L, Li Z, Cao Y, Fan D, Chui D, Guo X (2016) Increased extrasynaptic GluN2B expression is involved in cognitive impairment after isoflurane anesthesia. Exp Ther Med 12(1):161-168. https://doi.org/10.3892/etm.2016.3306

25. Gong Q-H, Wang Q, Pan L-L, Liu X-H, Huang H, Zhu Y-Z (2010) Hydrogen sulfide attenuates lipopolysaccharide-induecd cognitive impairment: a proinflammatory pathway in rats, Pharmacol Biochem Behav 96(1):52-58. https://doi.org/10.1016/j.pbb.2010.04.006

26. Vedder LC, Smith CC, Flannigan AE, McMahon LL (2013) Estradiol-induced increase in novel object recognition requires hippocampal NR2B-containing NMDA receptors. Hippocampus 23(1):108-115. https://doi.org/10.1002/hipo.22068

27. Paxinos G, Watson C (2007) The rat brain in stereotaxic coordinates, sixth edn. Elesvier

28. Jiang CH, Lee S, Park IY, Song A, Moon C, Cho GW (2019) Memantine Attenuates Salicylate-induced Tinnitus Possibly by Reducing NR2B Expression in Auditory Cortex of Rat. Exp Neurobiol 28(4):495503. https://doi.org/10.5607/en.2019.28.4.495 
29. Barker GR, Bird F, Alexander V, Warburton EC (2007) Recognition memory for objects, place, and temporal order: a disconnection analysis of the role of the medial prefrontal cortex and perirhinal cortex. J Neurosci 27(11):2948-2957. https://doi.org/10.1523/JNEUROSCI.5289-06.2007

30. Barker GR, Warburton EC (2011) When is the hippocampus involved in recognition memory? J Neurosci 31(29):10721-10731. https://doi.org/10.1523/JNEUROSCI.6413-10.2011

31. Chen B, Deng X, Wang B, Liu H (2016) Etanercept, an inhibitor of TNF-a, prevents propofol-induced neurotoxicity in the developing brain. Int J Dev Neurosci 55:91-100.

https://doi.org/10.1016/j.ijdevneu.2016.10.002

32. Chen B, Deng X, Wang B, Liu H (2016) Persistent neuronal apoptosis and synaptic loss induced by multiple but not single exposure of propofol contribute to long-term cognitive dysfunction in neonatal rats. J Toxicol Sci 41(5):627-636. https://doi.org/10.2131/jts.41.627

33. Zhang Z, Song Z, Shen F, Xie P, Wang J, Zhu A, Zhu G (2020) Ginsenoside Rg1 prevents PTSD-like behaviors in mice through promoting synaptic proteins, reducing Kir4.1 and TNF-a in the hippocampus. Mol Neurobiol. https://doi.org/10.1007/s12035-020-02213-9

34. Huttner WB, Schiebler W, Greengard P, Camilli P De (1983) Synapsin-I (Protein-I), a nerve terminalspecific phosphoprotein .III. Its association with synaptic vesicles studied in a highly purified synaptic vesicle preparation. J Cell Biol 96(5):1374-1388. https://doi.org/10.1083/jcb.96.5.1374

35. Carlin RK, Grab DJ, Cohen RS, Siekevitz P (1980) Isolation and characterization of postsynaptic densities from various brain regions: enrichment of different types of postsynaptic densities. $J$ Cell Biol 86(3):831-845. https://doi.org/10.1083/jcb.86.3.831

36. Smith KE, Gibson ES, Dell'Acqua ML (2006) cAMP-dependent protein kinase postsynaptic localization regulated by NMDA receptor activation through translocation of an A-kinase anchoring protein scaffold protein. J Neurosci 26(9):2391-2402. https://doi.org/10.1523/JNEUROSCI.309205.2006

37. Matus Al, Taff-Jones DH (1978) Morphology and molecular composition of isolated postsynaptic junctional structures. Proc R Soc Lond B Biol Sci 203(1151):135-151.

https://doi.org/10.1098/rspb.1978.0097

38. Philips GR, Huang JK, Wang Y, Tanaka H, Shapiro L, Zhang W, et al (2001) The presynaptic particle web: ultrastructure, composition, dissolution, and reconstitution. Neuron 32(1):63-

77. https://doi.org/10.1016/s0896-6273(01)00450-0

39. Milnerwood AJ, Gladding CM, Pouladi MA, Kaufman AM, Hines RM, Boyd JD, et al (2010) Early increase in extrasynaptic NMDA receptor signaling and expression contributes to phenotype onset in Huntington's disease mice. Neuron 65(2):178-190. https://doi.org/10.1016/j.neuron.2010.01.008

40. Deng X, Chen B, Wang B, Zhang J, Liu H (2017) TNF-a mediates the intrinsic and extrinsic pathway in propofol-induced neuronal apoptosis via PI3K/Akt signaling pathway in rat prefrontal cortical neurons. Neurotox Res 32(3):409-419. https://doi.org/10.1007/s12640-017-9751-8

41. Shi D-D, Huang Y-H, Lai CSW, Dong CM, Ho LC, Li X-Y, et al (2019) Ginsenoside Rg1 prevents chemotherapy-induced cognitive impairment: Associations with microglia-mediated cytokines, 
neuroinflammation, and neuroplasticity. Mol Neurobiol 56:5626-5642.

https://doi.org/10.1007/s12035-019-1474-9

42. Zarifkar A, Choopani S, Ghasemi R, Naghdi N, Maghsoudi AH, Maghsoudi N, et al (2010) Agmatine prevents LPS-induced spatial memory impairment and hippocampal apoptosis. Eur J Pharmacol 634(1-3):84-88. https://doi.org/10.1016/j.ejphar.2010.02.029

43. Feng Y, Xue H, Zhu J, Yang L, Zhang F, Qian R, et al (2016) ESE1 is associated with neuronal apoptosis in lipopolysaccharide induced neuroinflammation. Neurochem Res 41(10):2752-2762. https://doi.org/10.1007/s11064-016-1990-1

44. Wang K-C, Fan L-W, Kaizaki A, Pang Y, Cai Z, Tien L-T (2013) Neonatal lipopolysaccharide exposure induces long-lasting learning impairment, less anxiety-like response and hippocampal injury in adult rats. Neuroscience 234:146-157. https://doi.org/10.1016/j.neuroscience.2012.12.049

45. Lu W, Roche KW (2012) Posttranslational regulation of AMPA receptor trafficking and function. Curr Opin Neurobiol 22(3):470-479. https://doi.org/10.1016/j.conb.2011.09.008

46. Monyer H, Burnashev N, Laurie DJ, Sakmann B, Seeburg PH (1994) Developmental and regional expression in the rat brain and functional properties of four NMDA receptors. Neuron 12(3):529-540. https://doi.org/10.1016/0896-6273(94)90210-0

47. Parsons MP, Raymond LA (2014) Extrasynaptic NMDA receptor involvement in central nervous system disorders. Neuron 82(2):279-293. https://doi.org/10.1016/j.neuron.2014.03.030

48. Varbanov H, Dityatev A (2017) Regulation of extrasynaptic signaling by polysialylated NCAM: impact for synaptic plasticity and cognitive function. Mol Cell Neurosci 81:12-21.

https://doi.org/10.1016/j.mcn.2016.11.005

49. Goebel-Goody SM, Davies KD, Alvestad Linger RM, Freund RK, Browning MD (2009) Phosphoregulation of synaptic and extrasynaptic $\mathrm{N}$-methyl-d-aspartate receptors in adult hippocampal slices. Neuroscience 158(4):1446-1459. https://doi.org/10.1016/j.neuroscience.2008.11.006

50. Stazi M, Wirths $O$ (2021) Chronic memantine treatment ameliorates behavioral deficits, neuron loss, and impaired neurogenesis in a model of Alzheimer's disease. Mol Neurobiol 58(1):204-216. https://doi.org/10.1007/s12035-020-02120-z

51. Fisher G, Mutel V, Trube G, Malherbe P, Kew JN, Mohasci E, et al (1997) Ro25-6981, a highly potent and selective blocker of N-methyl-D-aspartate receptors containing the NR2B subunit. Characterization in vitro. J Pharmacol Exp Ther 283(3):1285-1292.

52. Zarbato GF, de Souza Goldim MP, Giustina AD, Danielski LG, Mathias K, Florentino D, et al (2018) Dimethyl fumarate limits neuroinflammation and oxidative stress and improves cognitive impairment after polymicrobial sepsis, Neurotox Res 34(3):418-430. https://doi.org/10.1007/s12640-018-9900-8

53. Safavynia SA, Goldstein PA (2019) The role of neuroinflammation in postoperative cognitive dysfunction: moving from hypothesis to treatment. Front Psychiatry 9:752. https://doi.org/10.3389/fpsyt.2018.00752 
54. Tripathi A, Paliwal P, Krishnamurthy S (2017) Piracetam attenuates LPS-induced neuroinflammation and cognitive impairment in rats. Cell Mol Neurobiol 37(8):1373-1386.

https://doi.org/10.1007/s10571-017-0468-2

55. Belarbi K, Jopson T, Tweedie D, Arellano C, Luo W, Greig NH, et al (2012) TNF-a protein synthesis inhibitor restores neuronal function and reverses cognitive deficits induced by chronic neuroinflammation. J Neuroinflammation 9:23. https://doi.org/10.1186/1742-2094-9-23

56. Cholvin T, Loureiro M, Cassel R, Cosquer B, Herbeaux K, de Vasconcelos AP, et al (2016) Dorsal hippocampus and medial prefrontal cortex each contribute to the retrieval of a recent spatial memory in rats. Brain Struct Funct 221(1):91-102. https://doi.org/10.1007/s00429-014-0894-6

57. Ennaceur A, Neave N, Aggleton JP (1996) Neurotoxic lesions of the perirhinal cortex do not mimic the behavioural effects of fornix transection in the rat. Behav Brain Res 80(1-2):9-25. https://doi.org/10.1016/0166-4328(96)00006-x

58. Hannesson DK, Vacca G, Howland JG, Phillips AG (2004) Medial prefrontal cortex is involved in spatial temporal order memory but not spatial recognition memory in tests relying on spontaneous exploration in cortex. Psychopharmacology (Berl) 232(24):4433-4444. https://doi.org/10.1007/s00213-015-4071-2

59. Tosaka S, Tosaka R, Matsumoto S, Maekawa T, Cho S, Sumikawa K (2011) Roles of cyclooxygenase 2 in sevoflurane- and olprinone-induced early phase of preconditioning and postconditioning against myocardial infarction in rat hearts. J Cardiovasc Pharmacol Ther 16(1):72-78. https://doi.org/10.1177/1074248410380208

60. Hu X, Wang J, Zhang Q, Duan X, Chen Z, Zhang Y (2016) Postconditioning with sevoflurane amileorates spatial learning and memory deficit after hemorrhage shock and resuscitation in rats. $J$ Surg Res 206(2):307-315. https://doi.org/10.1016/j.jss.2016.08.026

61. Luo C, Zhang YL, Luo W, Zhou FH, Li CQ, Xu JM, et al (2015) Differential effects of general anesthetics on anxiety-like behavior in formalin-induced pain: involvement of ERK activation in the anterior cingulate

62. Schoen J, Husemann L, Tiemeyer C, Lueloh A, Sedemund-Adib B, Berger KU, et al (2011) Cognitive function after sevoflurane- vs propofol-based anaesthesia for on-pump cardiac surgery: a randomized controlled trial. Br J Anaesth 106(6):840-850. https://doi.org/10.1093/bja/aer091 rats. Behav Brain Res 153(1):273-283. https://doi.org/10.1016/j.bbr.2003.12.004

63. Hardingham GE, Bading H (2003) The Yin and Yang of NMDA receptor signalling. Trends Neurosci 26(2):81-89. https://doi.org/10.1016/S0166-2236(02)00040-1

64. Warburton EC, Barker GR, Brown MW (2013) Investigations into the involvement of NMDA mechanisms in recognition memory. Neuropharmacology 74(100):41-47. https://doi.org/10.1016/j.neuropharm.2013.04.013

65. Réus GZ, Valvassori SS, Machado RA, Martins MR, Gavioli EC, Quevedo J (2007) Acute treatment with low doses of memantine does not impair aversive, non-associative and recognition memory in 
rats. Naunyn Schmiedebergs Arch Pharmacol 376(5):295-300. https://doi.org/10.1007/s00210-0070235-x

66. Danysz W, Parsons CG (2002) Neuroprotective potential of ionotropic glutamate receptor antagonists. Neurotox Res 4(2):119-126. https://doi.org/10.1080/10298420290015872

67. Wu Q, Zheng R, Srisai D, McKnight GS, Palmiter RD (2013) NR2B subunit of the NMDA glutamate receptor regulates appetite in the parabrachial nucleus, Proc Natl Acad Sci USA 110(36):1476514770. https://doi.org/10.1073/pnas. 1314137110

68. Jiang M, Zhang W, Ma Z, Gu X (2013) Antinociception and prevention of hyperalgesia by intrathecal administration of Ro 25-6981, a highly selective antagonist of the 2B subunit of N-methyl-Daspartate receptor. Pharmacol Biochem Behav 112:56-63. https://doi.org/10.1016/j.pbb.2013.09.007

69. Zhan Y, Xia J, Wang X (2020) Effects of glutamate-related drugs on anxiety and compulsive behavior in rats with obsessive-compulsive disorder. Int J Neurosci 130(6):551-560. https://doi.org/10.1080/00207454.2019.1684276

70. Chen Y, Chen A, Luo X, Guo L, Tang Y, Bao C, et al (2014) Hippocampal NR2B-containing NMDA receptors enhance long-term potentiation in rats with chronic visceral pain. Brain Res 1570:43-53. https://doi.org/10.1016/j.brainres.2014.05.001

71. Luciano-Jaramillo J, Sandoval-García F, Vázquez-Del Mercado M, Gutiérrez-Mercado YK, NavarroHernández RE, Martínez-García EA, et al (2019) Downregulation of hippocampal NR2A/2B subunits related to cognitive impairment in a pristane-induced lupus BALB/c mice. PLos One 14(9): e0217190. https://doi.org/10.1371/journal.pone.0217190

72. Chen D, Qi X, Zhuang R, Cao J, Xu Y, Huang X, et al (2019) Prenatal propofol exposure downregulates NMDA receptor expression and causes cognitive and emotional disorders in rats, Eur $J$ Pharmacol 843:268-276. https://doi.org/10.1016/j.ejphar.2018.11.032

73. Stabernack C, Sonner JM, Laster M, Zhang Y, Xing Y, Sharma M, et al (2003) Spinal N-methyl-daspartate receptors may contribute to the immobilizing action of isoflurane. Anesth Analg 96(1):102107. https://doi.org/10.1097/00000539-200301000-00022

74. Franks NP (2006) Molecular targets underlying general anaesthesia. Br J Pharmacol 147 Suppl 1(Suppl1):S72-81. https://doi.org/10.1038/sj.bjp.0706441

75. Gladding CM, Raymond LA (2011) Mechanisms underlying NMDA receptor synaptic/extrasynaptic distribution and function. Mol Cell Neurosci 48(4):308-320. https://doi.org/10.1016/j.mcn.2011.05.001

76. Wang WY, Luo Y, Jia LJ, Hu SF, Lou XK, Shen SL, et al (2014) Inhibition of aberrant cyclin-dependent kinase 5 activity attenuates isoflurane neurotoxicity in the developing brain. Neuropharmacology 77:90-99. https://doi.org/10.1016/j.neuropharm.2013.09.006

77. Yang X, Zhang W, Wu H, Fu S, Yang J, Liu S, et al (2020) Downregulation of CDK5 restores sevoflurane-induced cognitive dysfunction by promoting SIRT1-mediated autophagy. Cell Mol Neurobiol 40(6):955-965. https://doi.org/10.1007/s10571-020-00786-6 
78. Zhang S, Edelmann L, Liu J, Crandall JE, Morabito MA (2008) Cdk5 regulates the phosphorylation of tyrosine 1472 NR2B and the surface expression of NMDA receptors. J Neurosci 28(2):415-424. https://doi.org/10.1523/JNEUROSCI.1900-07.2008

\section{Figures}

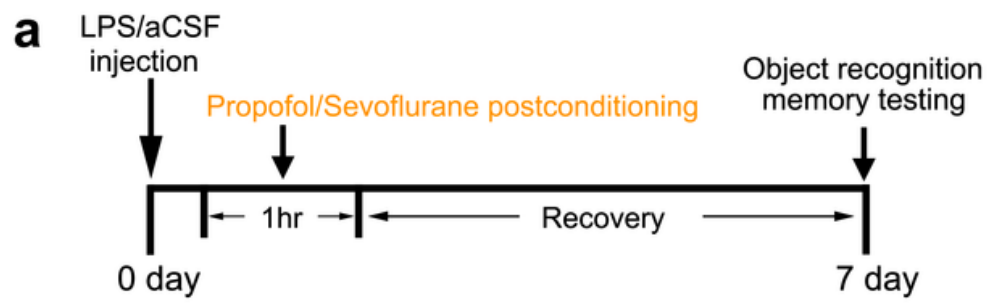

Novel object-place recognition

b Sample (5min)
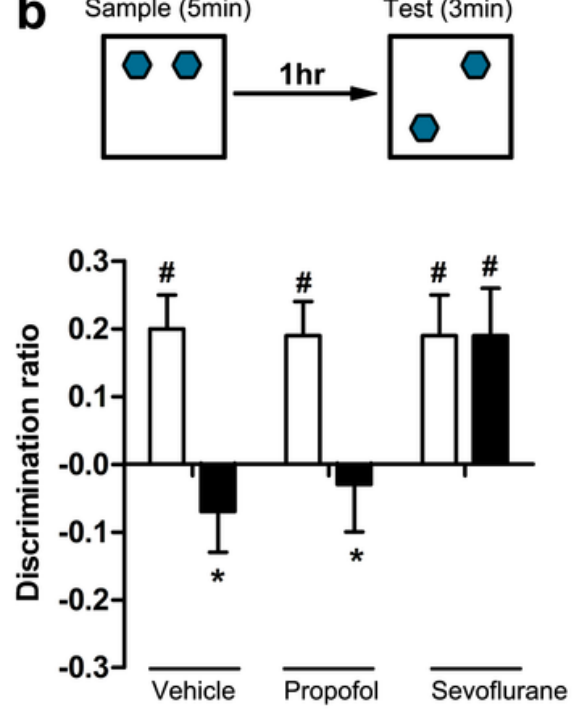

Novel object recognition

C
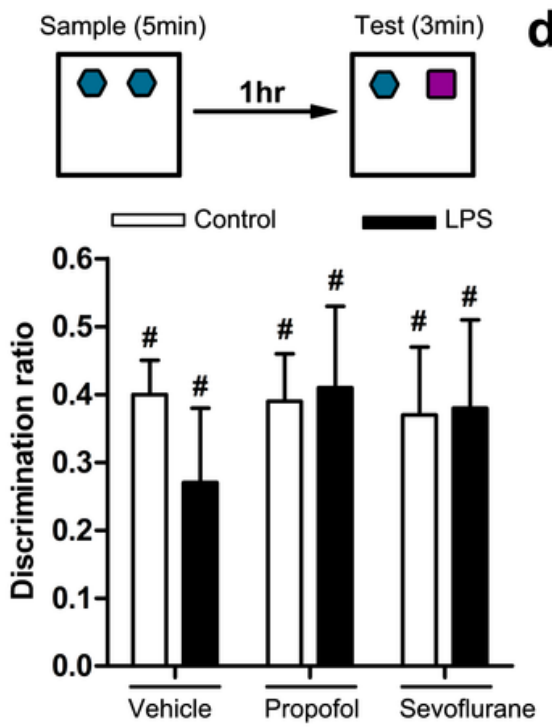

Temporal order memory

d Sample1 (5min) Sample2 (5min) Test (3min)
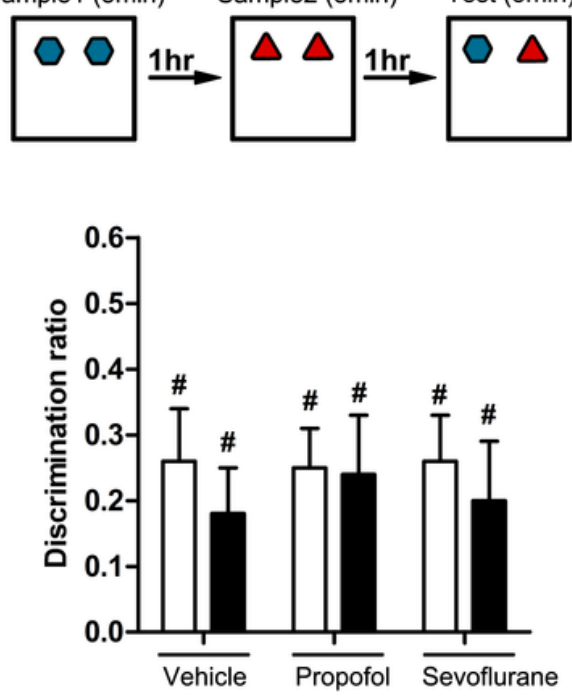

Figure 1

Object recognition memory tests after LPS injection and postconditioning with general anesthetics. (a) Schematic illustration of the experimental design. (b) Top: experimental procedure for novel object-place recognition memory. Bottom: the discrimination ratio was measured for control and LPS-injected rats post-treated with propofol or sevoflurane, or with vehicle only. (c) Top: experimental procedure for novel object recognition memory. Bottom: the discrimination ratio. (d) Top: experimental procedure for temporal order memory. Bottom: the discrimination ratio (Vehicle: $\mathrm{n}=10$ for Control and 9 for LPS; Propofol: $n=10$ for Control and 9 for LPS; Sevoflurane: $\mathrm{n}=10$ for Control and 10 for LPS). Data are expressed as mean \pm SEM, * $p<0.05$ vs. Control, \#p< 0.05 vs. zero. 
a

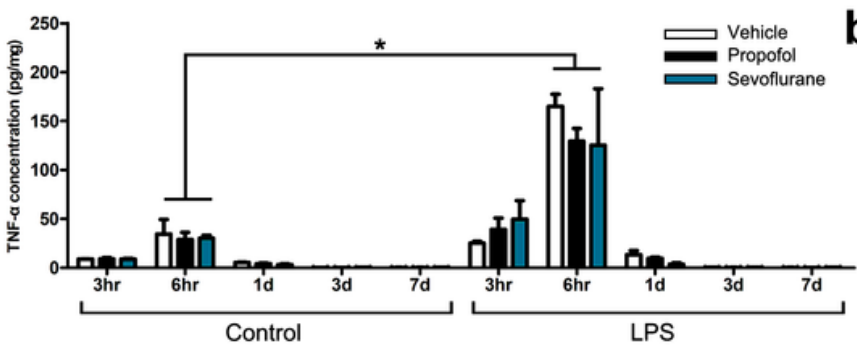

C

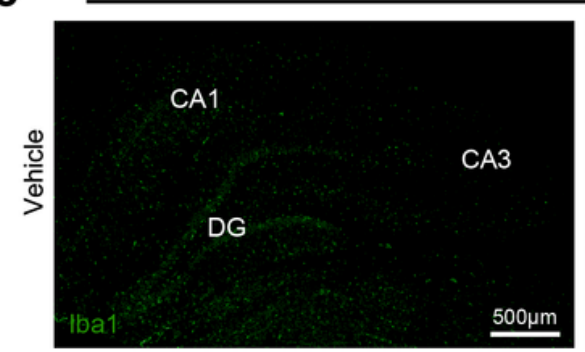

$\mathrm{CA} 1$

흥
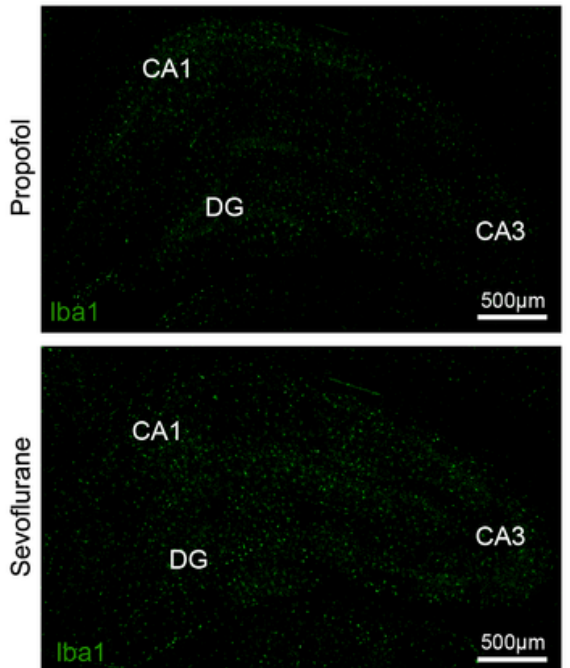

d

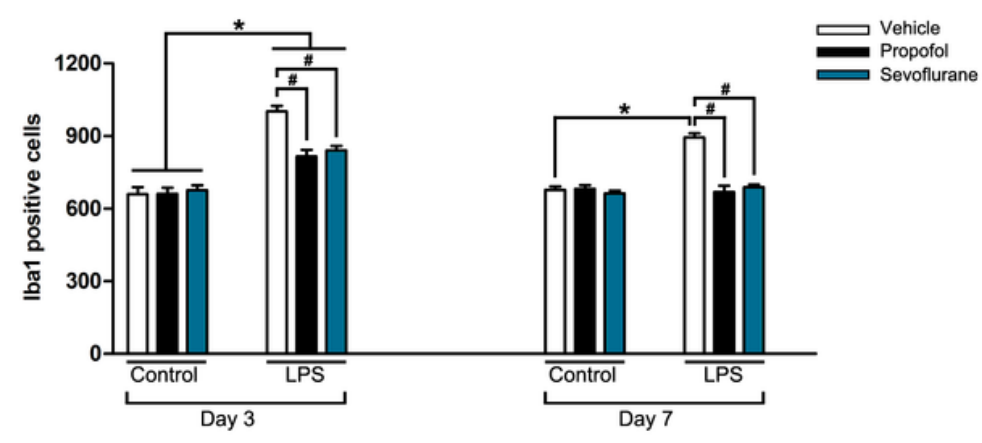

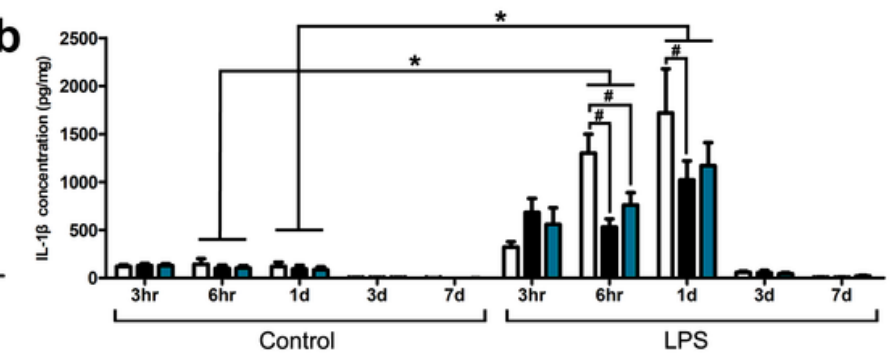

LPS
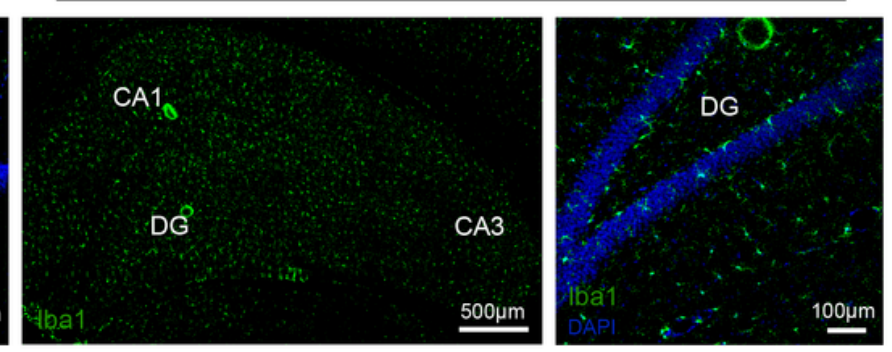

CA1
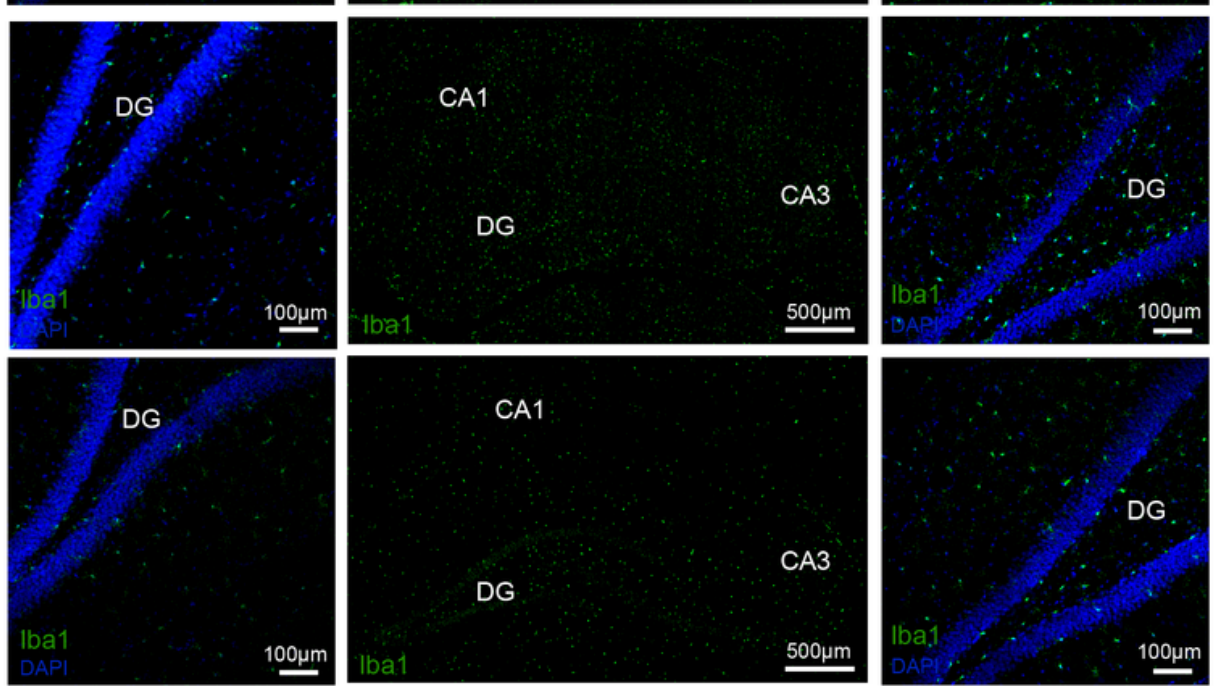

DG

$\mathrm{CA} 3$

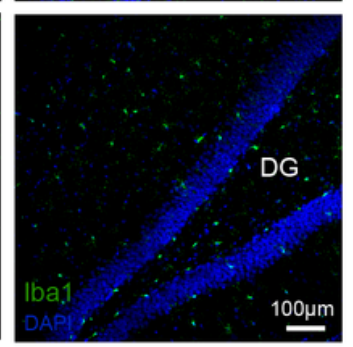

\section{Figure 2}

Expressional analysis of two major proinflammatory cytokines and number of microglia in the dHPC after LPS injection and postconditioning with general anesthetics. (a) TNF- $\alpha$ and (b) IL-1 $\beta$ concentrations in the dHPC were measured by ELISA at $3 \mathrm{hr}, 6 \mathrm{hr}$, and on day 1 , day 3 , and day 7 after LPS injection ( $\mathrm{n}=6$ per group). (c) Representative 10x objective images of immunofluorescence staining for iba1 (a microglia marker, green) in the dHPC on day 7 after LPS injection, DAPI staining in blue. (d) Quantitative analysis of iba $1+$ cells in the dHPC on day 3 and day 7 ( $n=6$ per group). Results are expressed as the mean \pm SEM. *p $<0.05$ vs. Control $₫ p<0.05$ vs. Vehicle. 
a

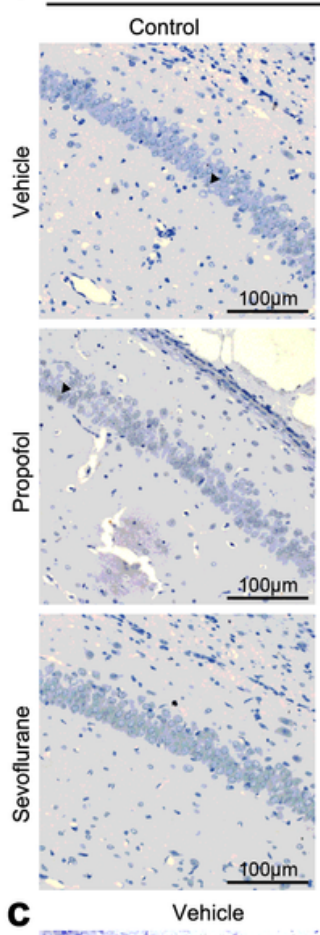

LPS

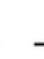

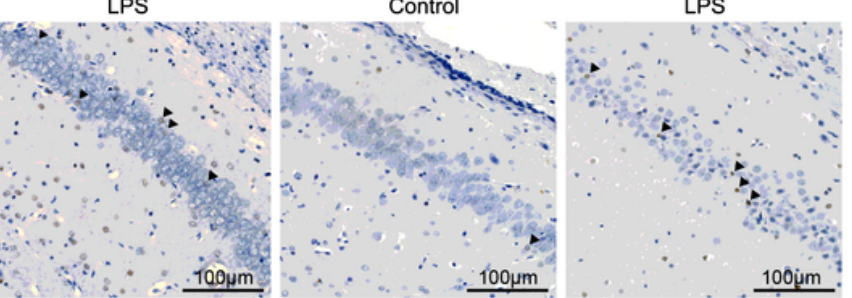

Day 7

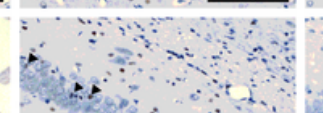

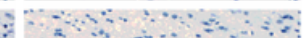
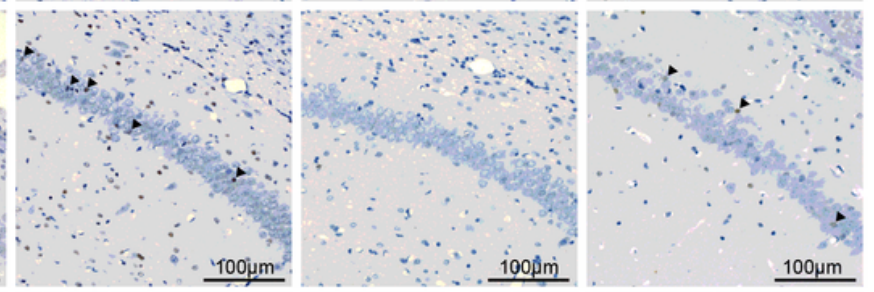

$100 \mu \mathrm{m}$
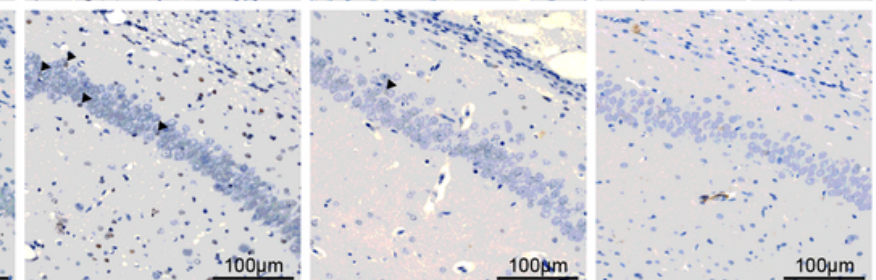

C
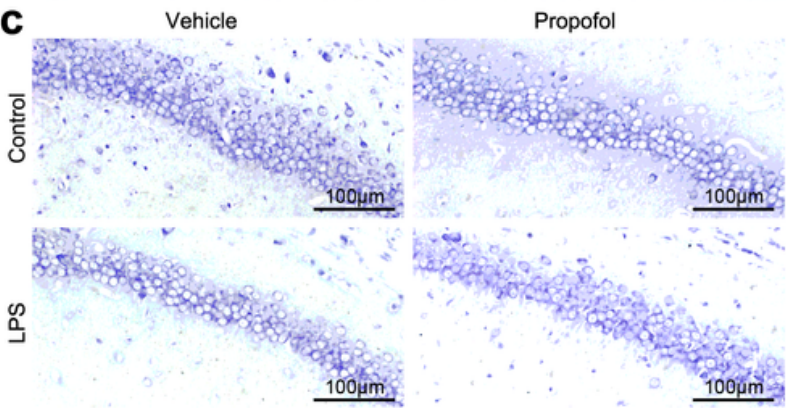

$100 \mathrm{sm}$

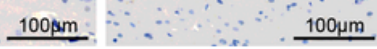
Sevoflurane

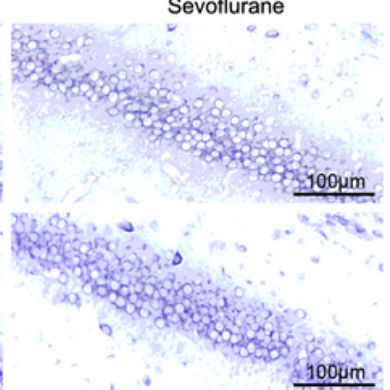

b

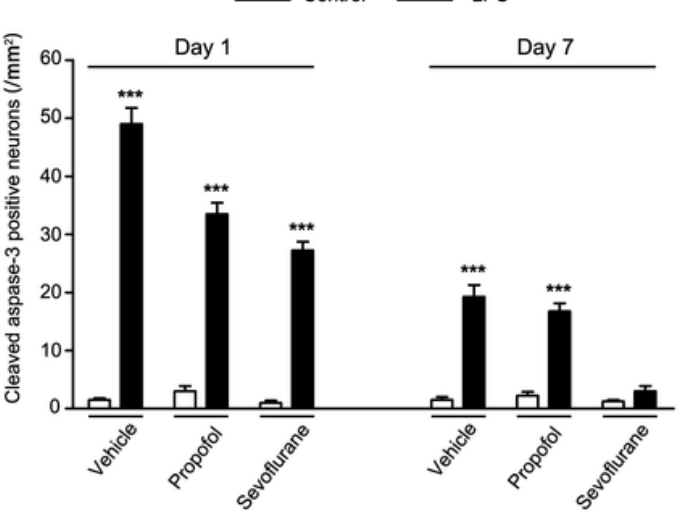

d

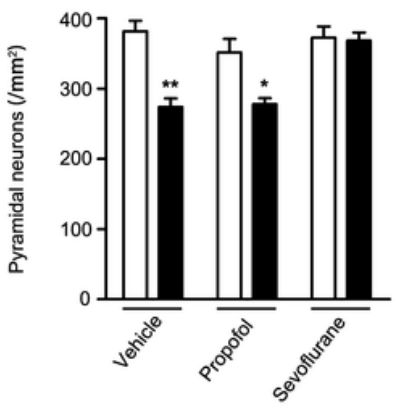

Figure 3

Expressional analysis of neuronal apoptosis and pyramidal neuron in the dorsal hippocampal CA1 region after LPS injection and postconditioning with general anesthetics. (a) Representative 20x objective images of immunohistochemistry staining for cleaved caspase-3 (an apoptosis marker, brown) in the dorsal hippocampal CA1 region on day 1 and day 7 after LPS injection. Black triangles indicate cleaved caspase-3+ neurons. (b) Quantitative analysis of the densities of cleaved caspase-3+ neurons in the dorsal hippocampal CA1 region on day 1 and day 7 ( $n=6$ per group). (c) Representative 20x objective images of Nissl staining for pyramidal neurons in the dorsal hippocampal CA1 region on day 7 after LPS injection. (d) Quantitative analysis of the pyramidal neuronal densities in the dorsal hippocampal CA1 region on day 7 ( $n=6$ per group). Results are expressed as the mean \pm SEM. ${ }^{\star} p<0.05, * \star p<0.01$, and ${ }^{* \star \star} p$ $<0.001$ vs. Control. 

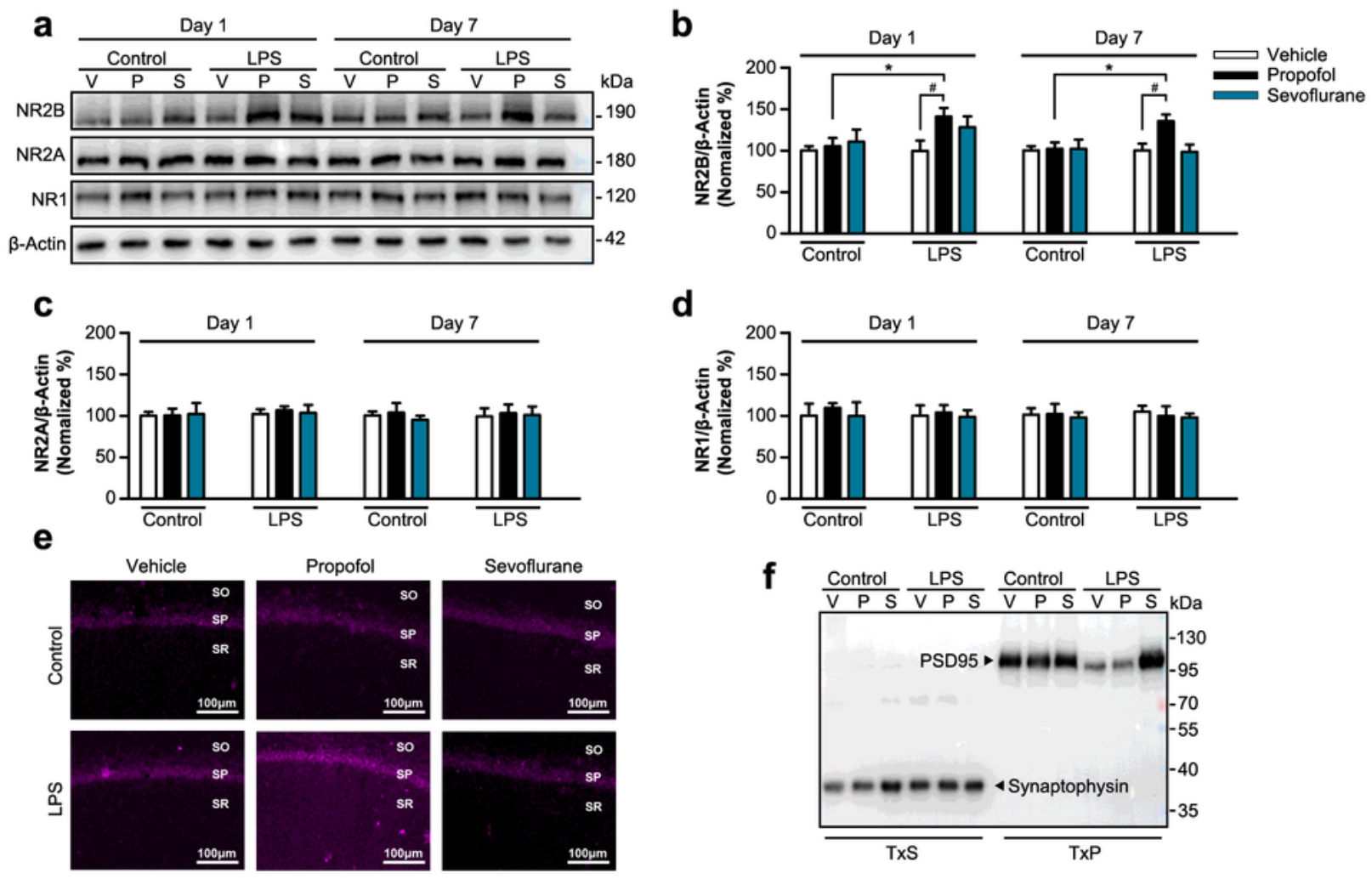

d
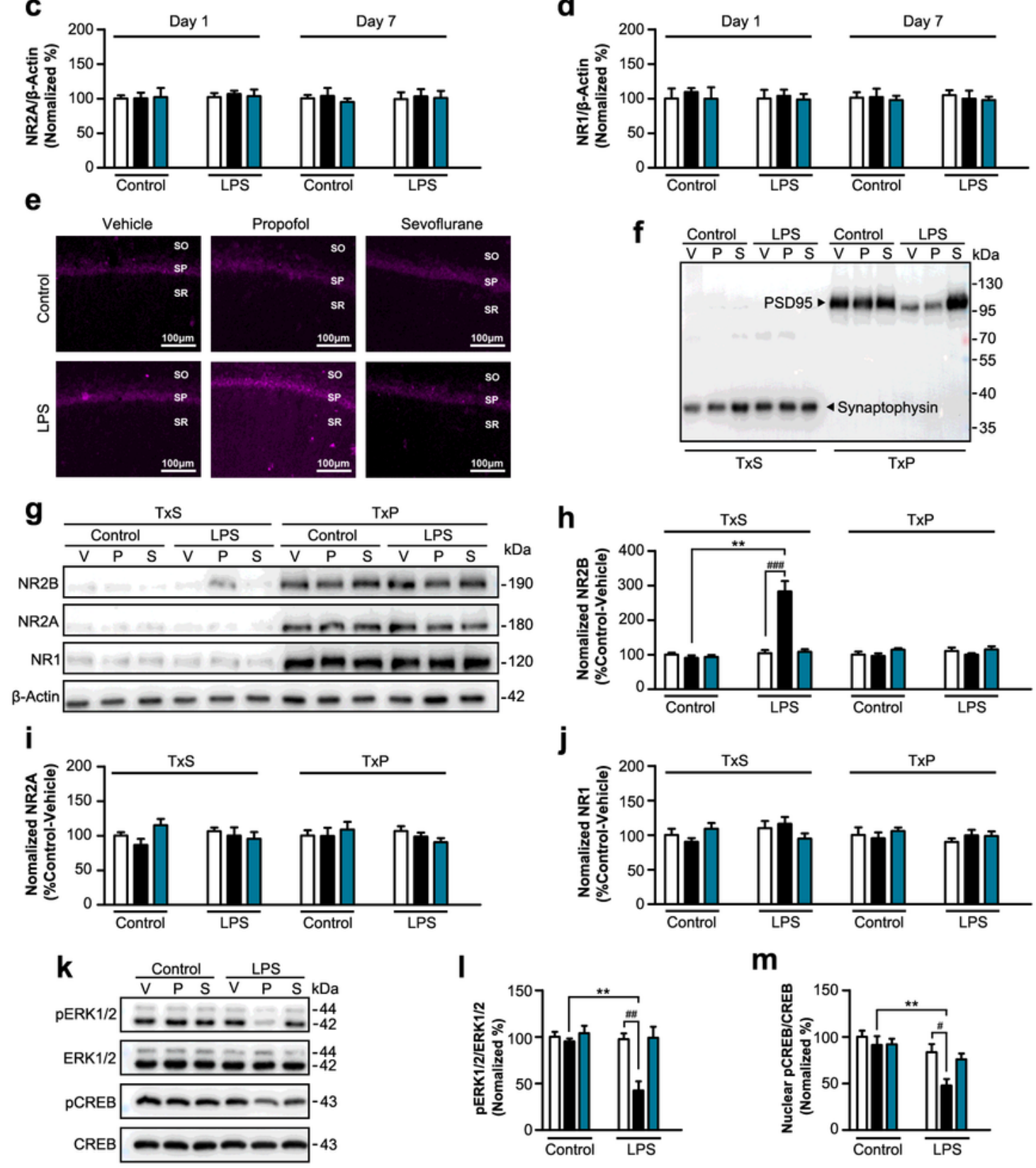

m

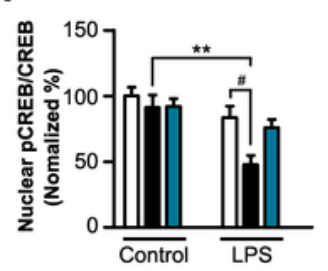

\section{Figure 4}

Expressional analysis of NMDA receptor subunits and signaling pathway downstream molecules in the dHPC after LPS injection and postconditioning with general anesthetics. (a) Representative immunoblots of NR2B, NR2A, and NR1 proteins in the dHPC on day 1 and day 7 after LPS injection. (b-d) Densitometric analysis of NR2B, NR2A, and NR1 proteins in the dHPC on day 1 and day 7 ( $n=6$ per group). (e) Representative 10x objective images of immunofluorescence staining for NR2B (purple) on day 7 in the 
dHPC. SO stratum oriens, SP stratum pyramidale, SR stratum radiatum. (f) Representative immunoblots of PSD95 and synaptophysin showing the efficiency of separating TxS (extrasynaptic) and TxP (synaptic) fractions in the dHPC. TxS Triton X-100 soluble, TxP Triton X-100 insoluble. (g) Representative immunoblots of NR2B, NR2A, and NR1 proteins in the TxS and TxP of dHPC on day 7 after LPS injection. (h-j) Densitometric analysis of NR2A, NR2B, and NR1 proteins in the TxS and TxP of dHPC on day 7 ( $n=6$ per group). (k) Representative immunoblots of phospho-ERK1/2, ERK1/2 in the total dHPC homogenate and phospho-CREB, CREB in the nuclear fraction of dHPC on day 7 after LPS injection. (I and $\mathrm{m}$ ) Densitometric analysis of phospho-ERK1/2, ERK $1 / 2$ in the total dHPC homogenate and phospho-CREB, CREB in the nuclear fraction of $d H P C$ on day 7 ( $n=6$ per group). $V$ vehicle, $P$ propofol $S$ sevoflurane. Data are presented as mean \pm SEM. ${ }^{* *} p<0.01$ vs. Control, $\# p<0.05$, \#\#p<0.01 vs. Vehicle.
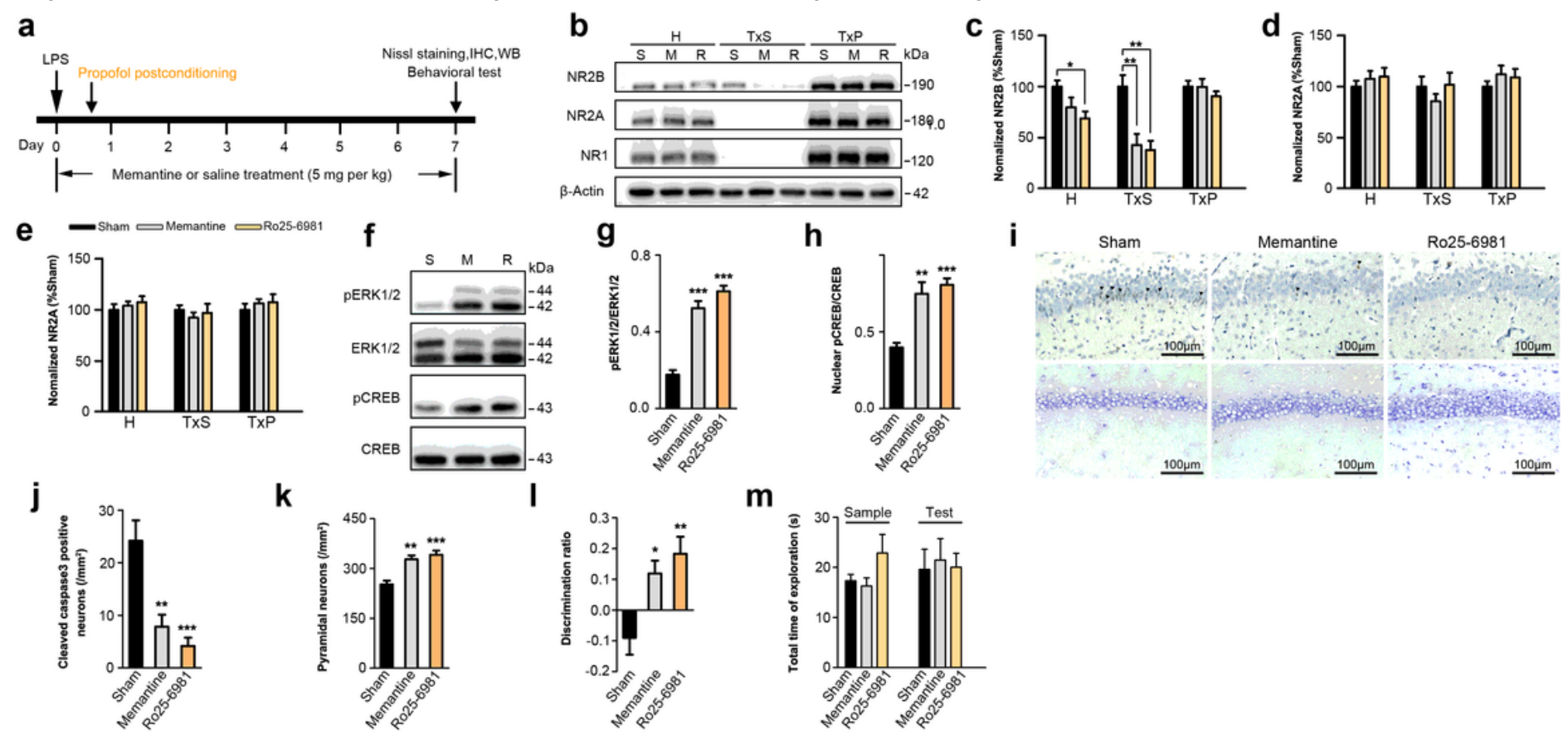

k
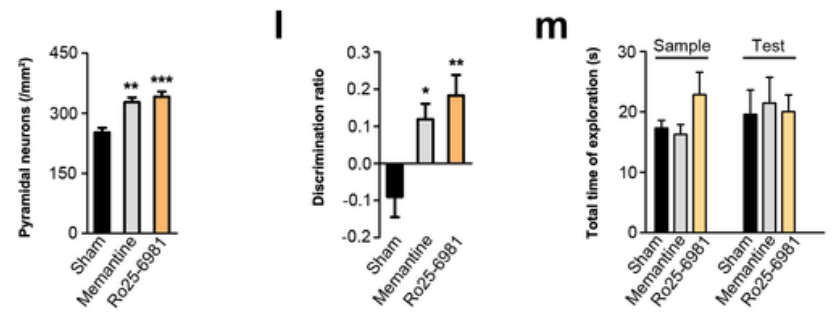

\section{Figure 5}

Figure captions Fig.1 Object recognition memory tests after LPS injection and postconditioning with general anesthetics. (a) Schematic illustration of the experimental design. (b) Top: experimental procedure for novel object-place recognition memory. Bottom: the discrimination ratio was measured for control and LPS-injected rats post-treated with propofol or sevoflurane, or with vehicle only. (c) Top: experimental procedure for novel object recognition memory. Bottom: the discrimination ratio. (d) Top: experimental procedure for temporal order memory. Bottom: the discrimination ratio (Vehicle: $n=10$ for Control and 9 for LPS; Propofol: $n=10$ for Control and 9 for LPS; Sevoflurane: $n=10$ for Control and 10 for LPS). Data are expressed as mean $\pm S E M,{ }^{*} p<0.05$ vs. Control, $\# p<0.05$ vs. zero. Fig.2. Expressional analysis of two major proinflammatory cytokines and number of microglia in the dHPC after LPS injection and postconditioning with general anesthetics. (a) TNF- $a$ and (b) IL-1 $\beta$ concentrations in the dHPC were measured by ELISA at $3 \mathrm{hr}, 6 \mathrm{hr}$, and on day 1 , day 3 , and day 7 after LPS injection ( $\mathrm{n}=6$ per group). (c) Representative 10x objective images of immunofluorescence staining for iba1 (a microglia marker, green) in the dHPC on day 7 after LPS injection, DAPI staining in blue. (d) Quantitative analysis of 
iba $1+$ cells in the dHPC on day 3 and day 7 ( $n=6$ per group). Results are expressed as the mean \pm SEM. *p $<0.05$ vs. Control凤\#p $<0.05$ vs. Vehicle. Fig3. Expressional analysis of neuronal apoptosis and pyramidal neuron in the dorsal hippocampal CA1 region after LPS injection and postconditioning with general anesthetics. (a) Representative 20x objective images of immunohistochemistry staining for cleaved caspase-3 (an apoptosis marker, brown) in the dorsal hippocampal CA1 region on day 1 and day 7 after LPS injection. Black triangles indicate cleaved caspase-3+ neurons. (b) Quantitative analysis of the densities of cleaved caspase- $3+$ neurons in the dorsal hippocampal CA1 region on day 1 and day 7 ( $n=6$ per group). (c) Representative 20x objective images of Nissl staining for pyramidal neurons in the dorsal hippocampal CA1 region on day 7 after LPS injection. (d) Quantitative analysis of the pyramidal neuronal densities in the dorsal hippocampal CA1 region on day 7 ( $n=6$ per group). Results are expressed as the mean \pm SEM. ${ }^{*} p<0.05,{ }^{*} p<0.01$, and ${ }^{* \star *} p<0.001$ vs. Control. Fig4. Expressional analysis of NMDA receptor subunits and signaling pathway downstream molecules in the dHPC after LPS injection and postconditioning with general anesthetics. (a) Representative immunoblots of NR2B, NR2A, and NR1 proteins in the dHPC on day 1 and day 7 after LPS injection. (b-d) Densitometric analysis of NR2B, NR2A, and NR1 proteins in the dHPC on day 1 and day 7 ( $n=6$ per group). (e) Representative 10x objective images of immunofluorescence staining for NR2B (purple) on day 7 in the dHPC. SO stratum oriens, SP stratum pyramidale, SR stratum radiatum. (f) Representative immunoblots of PSD95 and synaptophysin showing the efficiency of separating TxS (extrasynaptic) and TxP (synaptic) fractions in the dHPC. TxS Triton X-100 soluble, TxP Triton X-100 insoluble. (g) Representative immunoblots of NR2B, NR2A, and NR1 proteins in the TxS and TxP of dHPC on day 7 after LPS injection. (h-j) Densitometric analysis of NR2A, NR2B, and NR1 proteins in the TxS and TxP of dHPC on day 7 ( $n=6$ per group). ( $k$ ) Representative immunoblots of phospho-ERK1/2, ERK1/2 in the total dHPC homogenate and phospho-CREB, CREB in the nuclear fraction of dHPC on day 7 after LPS injection. ( $\mathrm{l}$ and $\mathrm{m}$ ) Densitometric analysis of phosphoERK1/2, ERK1/2 in the total dHPC homogenate and phospho-CREB, CREB in the nuclear fraction of dHPC on day 7 ( $n=6$ per group). $V$ vehicle, $P$ propofol $S$ sevoflurane. Data are presented as mean $\pm S E M$. ${ }^{* *} p<$ 0.01 vs. Control, $\# p<0.05, \# \# p<0.01$ vs. Vehicle. Fig. 5 The role of NR2B subunit in propofol postconditioning-induced neurotoxicity after LPS injection. (a) Schematic illustration of the experimental procedure. Rats were treated with i.p. memantine $(5 \mathrm{mg} / \mathrm{kg})$ or intrahippocampal injection of Ro25-6981 $(0.375 \mu \mathrm{g})$ after propofol postconditioning following LPS injection for consecutive 7 days. After finishing the behavioral test, rats were sacrificed for Nissl staining, immunohistochemistry, and western blot. IHC immunohistochemistry, WB western blot. (b) Representative immunoblots of NR2B, NR2A, and NR1 proteins in the total dHPC homogenate, TxS (extrasynaptic), and TxP (synaptic) fractions respectively. (ce) Densitometric analysis of NR2B, NR2A, and NR1 proteins in total dHPC homogenate, TxS (extrasynaptic), and TxP (synaptic) fractions respectively ( $n=6$ per group). H homogenate, TxS Triton X100 soluble, TxP Triton X-100 insoluble, S sham, M memantine, R Ro25-6981. (f) Representative immunoblots of phospho-ERK1/2, ERK $1 / 2$ in the total dHPC homogenate and phospho-CREB, CREB in the nuclear fraction of dHPC. ( $g$ and $h$ ) Densitometric analysis of phospho-ERK1/2, ERK1/2 in the total $\mathrm{dHPC}$ homogenate and phospho-CREB, CREB in the nulear fraction of dHPC ( $n=6$ per group). (i) Representative 20x objective images of immunohistochemistry staining for cleaved caspase-3 (upper, black triangles) and Nissl staining for pyramidal neurons (bottom) in the dorsal hippocampal CA1 region. 
( $\mathrm{j}$ and $\mathrm{k}$ ) Quantitative analysis of the densities of cleaved caspase-3+ neurons and the pyramidal neuronal densities in the dorsal hippocampal CA1 region ( $n=6$ per group). (I) The discrimination ratio in novel object-place recognition. $(\mathrm{m})$ Total time of object exploration in novel object-place recognition during the sample, and test phases. ( $n=10$ per group) Data are presented as mean $\pm S E M .{ }^{*} p<0.05,{ }^{*} p<$ $0.01,{ }^{* \star *} p<0.001$ vs. Sham.

\section{Supplementary Files}

This is a list of supplementary files associated with this preprint. Click to download.

- manuscriptSIMolNeurobiology.pdf 